\title{
Factors Affecting Marsh Vegetation at the Liberty Island Conservation Bank in the Cache Slough Region of the Sacramento-San Joaquin Delta, California
}

Open-File Report 2017-1077 
Front cover. North Little Holland Tract marsh.

Back top. U.S. Geological Survey personnel collecting samples at the Liberty Island Conservation Bank. Back bottom. Vegetation at the Liberty Island Conservation Bank. 


\section{Factors Affecting Marsh Vegetation at the Liberty Island Conservation Bank in the Cache Slough Region of the Sacramento- San Joaquin Delta, California}

By James L. Orlando and Judith Z. Drexler

Open-File Report 2017-1077 


\title{
U.S. Department of the Interior \\ RYAN K. ZINKE, Secretary
}

\section{U.S. Geological Survey William H. Werkheiser, Acting Director}

\author{
U.S. Geological Survey, Reston, Virginia: 2017
}

For more information on the USGS - the Federal source for science about the Earth, its natural and living resources, natural hazards, and the environment—visit http://www.usgs.gov or call 1-888-ASK-USGS.

For an overview of USGS information products, including maps, imagery, and publications, visit http://www.usgs.gov/pubprod/.

Any use of trade, firm, or product names is for descriptive purposes only and does not imply endorsement by the U.S. Government.

Although this information product, for the most part, is in the public domain, it also may contain copyrighted materials as noted in the text. Permission to reproduce copyrighted items must be secured from the copyright owner.

Suggested citation:

Orlando, J.L., and Drexler, J.Z., 2017, Factors affecting marsh vegetation at the Liberty Island Conservation Bank in the Cache Slough region of the Sacramento-San Joaquin Delta, California, 2017: U.S. Geological Survey Open-File Report 2017-1077, 25 p., https://doi.org/10.3133/ofr20171077.

ISSN 2331-1258 (online) 


\section{Acknowledgments}

The authors would like to thank Allegra Bukojemsky, Wildlands, Inc., for providing background information on the Liberty Island Conservation Bank.

The authors would also like to thank Jason Dunn, Andy Morita, Austyn Cromartie, Matt DeParsia, Corey Sanders, Megan McWayne, and Sean Stout of the U.S. Geological Survey for their help with field sampling and laboratory analyses during this project.

\section{Contents}

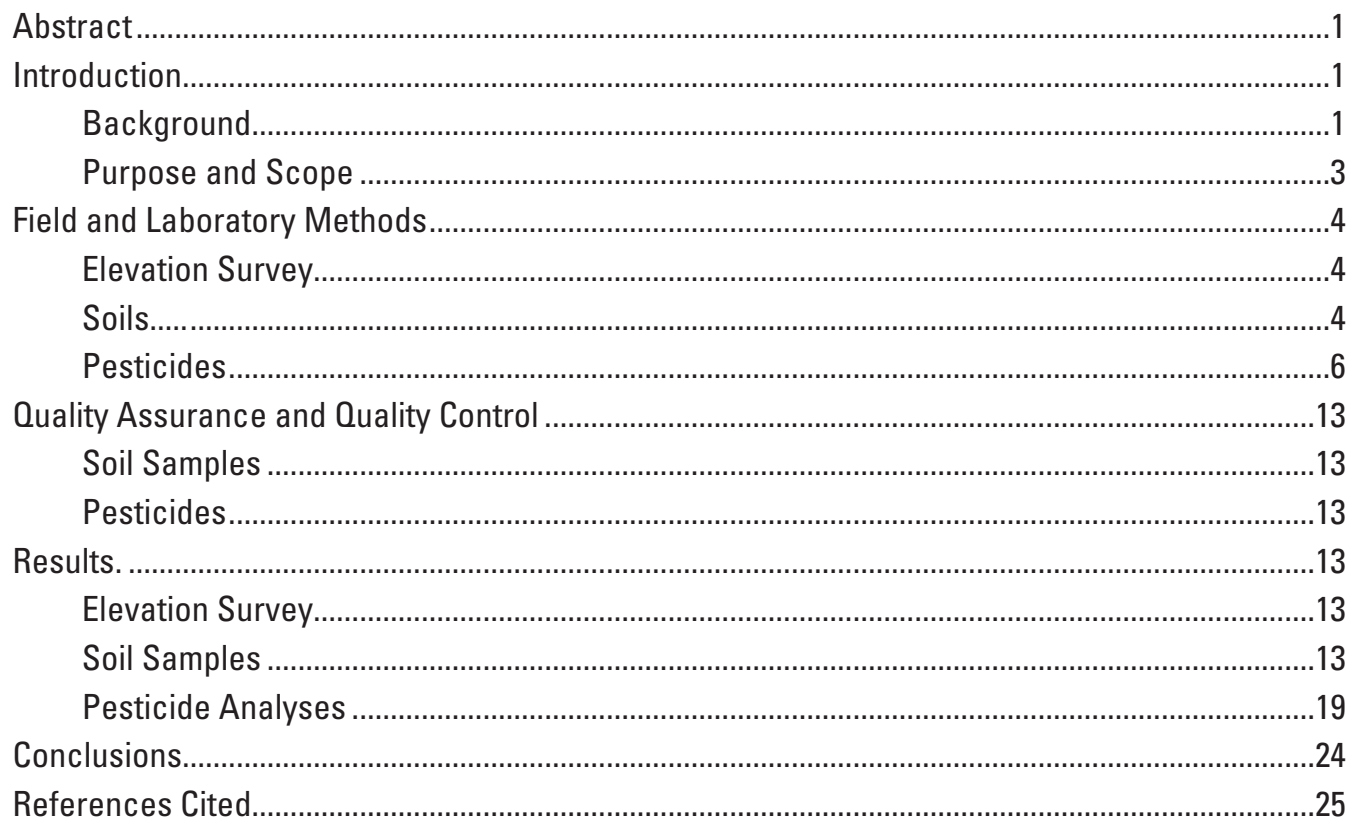




\section{Figures}

1. Aerial photograph showing marsh soil and water-quality sampling sites in the Cache Slough region of the Sacramento-San Joaquin Delta .....

2. Graph showing mean shoot length of California bulrush (Schoenoplectus californicus) collected from four marsh sites in the Cache Slough region of the Sacramento-San Joaquin Delta during September 2014.

3. Graph showing mean stem diameter of California bulrush collected from four marsh sites in the Cache Slough region of the Sacramento-San Joaquin Delta during September 2014

4. Map showing RTK-GPS (real-time kinematic global positioning system) surveypoint elevations, relative to the North American Vertical Datum of 1988, for two marsh sites in the Cache Slough region of the Sacramento-San Joaquin Delta, November 2015.

5. Graph showing mean weight as percentages of total carbon, organic carbon, total nitrogen, and total organic nitrogen measured in soil samples collected from four marsh sites in the Cache Slough region of the Sacramento-San Joaquin Delta, September 2014

6. Graph showing number of current-use pesticides detected at five surface-water sites in the Cache Slough region of the Sacramento-San Joaquin Delta, 2015.

\section{Tables}

1. Surface-water and soil sampling sites in the Cache Slough region of the Sacramento-San Joaquin Delta, California

2. Method detection limits for pesticides in water measured by the U.S. Geological Survey Organic Chemistry Research Laboratory.

3. Field water-quality parameter data measured at collection sites in $\mathbf{2 0 1 5}$ during water-sample collection in the Cache Slough region of the Sacramento-San Joaquin Delta, California

4. Elevation and coordinate data from the Global Positioning System survey at the north Little Holland Tract marsh site, November 2015.

5. Elevation and coordinate data from the Global Positioning System survey at the Liberty Island Conservation Bank marsh site, November 2015

6. Organic carbon and nitrogen measured in soil samples collected from marsh sites in the Cache Slough region of the Sacramento-San Joaquin Delta, September 2014

7. Pesticide concentrations measured in environmental water samples collected from the Cache Slough region of the Sacramento-San Joaquin Delta, California, 2015

8. Detection frequencies of pesticides in water samples collected from the Cache Slough region of the Sacramento-San Joaquin Delta, California, 2015. 


\section{Conversion Factors}

International System of Units to U.S. customary units

\begin{tabular}{lll}
\hline \multicolumn{1}{c}{ Multiply } & \multicolumn{1}{c}{ By } & \multicolumn{1}{c}{ To obtain } \\
\hline centimeter $(\mathrm{cm})$ & \multicolumn{1}{c}{ Length } \\
millimeter $(\mathrm{mm})$ & 0.3937 & inch (in.) \\
micrometer $(\mu \mathrm{m})$ & 0.03937 & inch (in.) \\
meter $(\mathrm{m})$ & 0.0003937 & inch (in.) \\
kilometer $(\mathrm{km})$ & 3.281 & foot (ft) \\
& 0.6214 & mile (mi) \\
\hline hectare $(\mathrm{ha})$ & \multicolumn{1}{c}{ Area } & acre \\
square meter $\left(\mathrm{m}^{2}\right)$ & $2.471 \quad$ Volume & acre \\
\hline liter $(\mathrm{L})$ & 0.0002471 & ounce, fluid (fl. oz) \\
liter $(\mathrm{L})$ & 33.81402 & gallon (gal) \\
liter $(\mathrm{L})$ & 0.2642 & cubic inch (in $\left.{ }^{3}\right)$ \\
\hline
\end{tabular}

Temperature in degrees Celsius $\left({ }^{\circ} \mathrm{C}\right)$ may be converted to degrees Fahrenheit $\left({ }^{\circ} \mathrm{F}\right)$ as follows:

$$
{ }^{\circ} \mathrm{F}=\left(1.8 \times{ }^{\circ} \mathrm{C}\right)+32 .
$$

\section{Datum}

Vertical coordinate information is referenced to the North American Vertical Datum of 1988 (NAVD 88).

Horizontal coordinate information is referenced to the North American Datum of 1983 (NAD 83).

\section{Supplemental Information}

Specific conductance is given in microsiemens per centimeter at 25 degrees Celsius $(\mu \mathrm{S} / \mathrm{cm}$ at $\left.25^{\circ} \mathrm{C}\right)$.

Concentrations of chemical constituents in water are given in either milligrams per liter (mg/L) or nanograms per liter (ng/L).

\section{Abbreviations}

$\begin{array}{ll}\text { 3,4-DCA } & \text { 3,4-dichlooraniline } \\ \text { ANOVA } & \text { analysis of variance } \\ \text { CUP } & \text { Current-use Pesticide } \\ \text { DCPMU } & \text { N-(3,4-Dichlorophenyl)-N'-methylurea } \\ \text { GC/MS } & \text { gas chromatograph mass spectrometer } \\ \text { LC/MS/MS } & \text { liquid chromatography tandem mass spectrometry } \\ \text { LICB } & \text { Liberty Island Conservation Bank } \\ \text { OCRL } & \text { Organic Chemistry Research Laboratory } \\ \text { OC } & \text { quality control } \\ \text { RTK-GPS } & \text { real-time kinematic global positioning system } \\ \text { USGS } & \text { U.S. Geological Survey }\end{array}$





\title{
Factors Affecting Marsh Vegetation at the Liberty Island Conservation Bank in the Cache Slough Region of the Sacramento-San Joaquin Delta, California
}

\author{
By James L. Orlando and Judith Z. Drexler
}

\section{Abstract}

The Liberty Island Conservation Bank (LICB) is a tidal freshwater marsh restored for the purpose of mitigating adverse effects on sensitive fish populations elsewhere in the region. The LICB was completed in 2012 and is in the northern Cache Slough region of the Sacramento-San Joaquin Delta. The wetland vegetation at the LICB is stunted and yellow-green in color (chlorotic) compared to nearby wetlands. A study was done to investigate three potential causes of the stunted and chlorotic vegetation: (1) improper grading of the marsh plain, (2) pesticide contamination from agricultural and urban inputs upstream from the site, (3) nitrogen-deficient soil, or some combination of these. Water samples were collected from channels at five sites, and soil samples were collected from four wetlands, including the LICB, during the summer of 2015. Real-time kinematic global positioning system (RTK-GPS) elevation surveys were completed at the LICB and north Little Holland Tract, a closely situated natural marsh that has similar hydrodynamics as the LICB, but contains healthy marsh vegetation.

The results showed no significant differences in carbon or nitrogen content in the surface soils or in pesticides in water among the sites. The elevation survey indicated that the mean elevation of the LICB was about 26 centimeters higher than that of the north Little Holland Tract marsh. Because marsh plain elevation largely determines the hydroperiod of a marsh, these results indicated that the LICB has a hydroperiod that differs from that of neighboring north Little Holland Tract marsh. This difference in hydroperiod contributed to the lower stature and decreased vigor of wetland vegetation at the LICB. Although the LICB cannot be regraded without great expense, it could be possible to reduce the sharp angle of the marsh edge to facilitate deeper and more frequent tidal flooding along the marsh periphery. Establishing optimal elevations for restored wetlands is necessary for obtaining the full suite of ecosystem services provided by tidal wetlands. A better system of tidal benchmarks throughout the delta is needed to help restoration practitioners correctly grade the elevation of newly restored wetlands.

\section{Introduction}

\section{Background}

The Liberty Island Conservation Bank (LICB) is in the Cache Slough region of the Sacramento-San Joaquin Delta (fig. 1). The project is a fish mitigation bank, which is a wetland area restored for the purpose of providing compensation as required by state or federal law for unavoidable, adverse effects on sensitive fish populations from projects in the region. It was created with two primary goals: (1) to preserve, restore, and enhance 67.2 hectares of wildlife habitat and (2) to provide improvements to flood capacity and levee stability (Wildlands, Inc., 2009). As part of the restoration, the project included grading of 21.8 hectares to construct channels (2.7 hectares), emergent marsh (13.8 hectares), and riparian habitat (1.1 hectares). In addition, 366 linear meters of levee were graded to sustain wetland vegetation (Wildlands, Inc. 2009). The project was completed in 2012 .

In August of 2014, we noticed that dominant wetland plants at the LICB were shorter than those in nearby natural marshes and were yellow, or "chlorotic," in contrast to the deep-green plants in neighboring marshes. In September of 2014, we compared the stem height and diameter of Schoenoplectus californicus (S. californicus, California bulrush), a common tidal marsh species throughout Cache Slough, in the LICB to that of bulrushes in three nearby marshes (north Little Holland Tract, south Little Holland Tract, and south Prospect; fig. 1). We collected five plants from each of three well-dispersed 1-square meter $\left(\mathrm{m}^{2}\right)$ plots in each of the three nearby marshes. Because of the extensive presence of Typha species (cattails) at the LICB, we could not find enough $S$. californicus to measure plant height and diameter in plots, so we measured plants along three transects through the marsh. We analyzed the stem length and diameter data using one-way analysis of variance (ANOVA) and did post hoc pairwise comparisons using the Bonferroni method. 


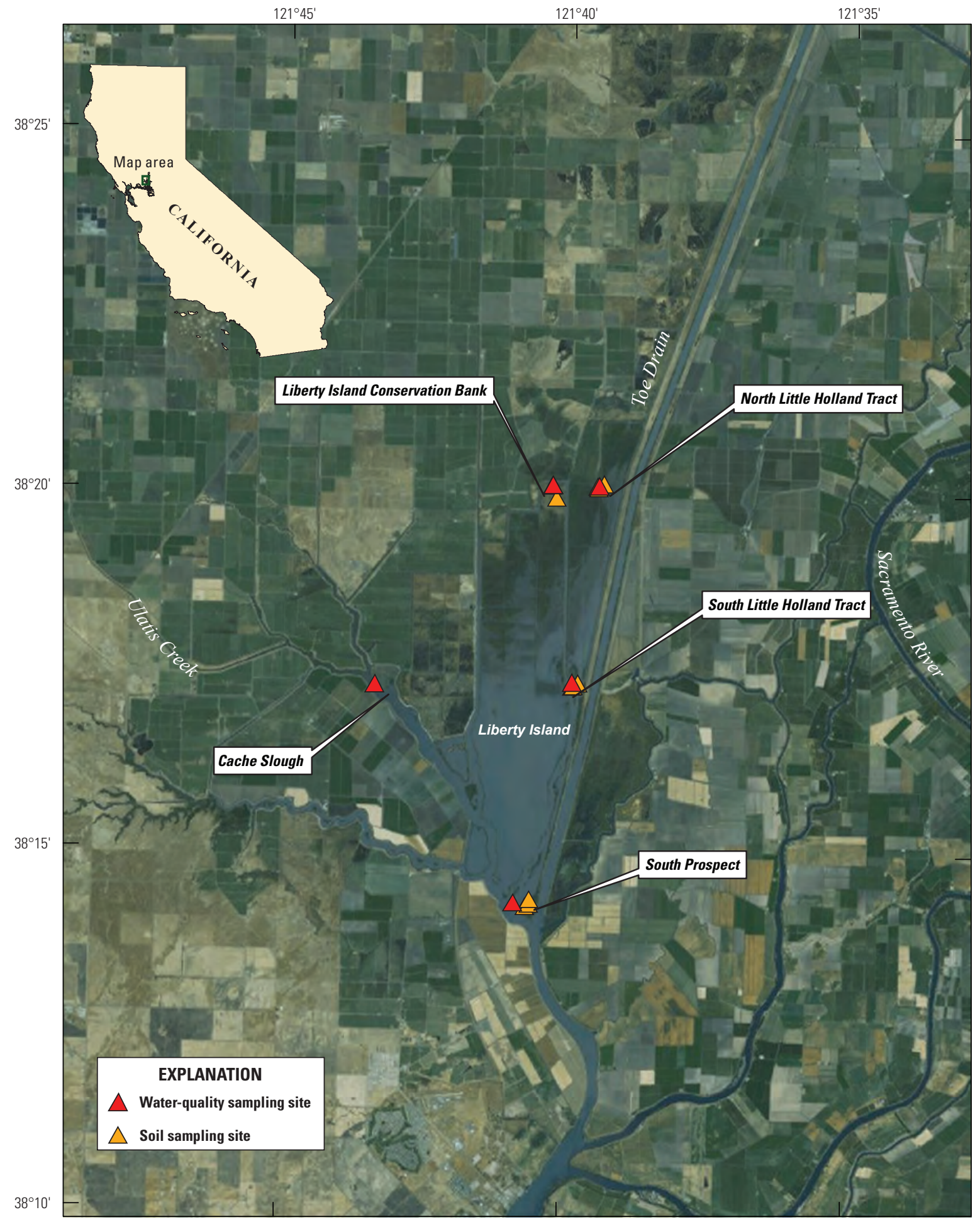

Base modified from U.S. Geological Survey and other Federal and State digital data, various scales; Albers Equal-Area Conic projection, standard parallels are $34^{\circ} 00^{\prime}$ and $40^{\circ} 30^{\prime}$; North American Datum of 1983

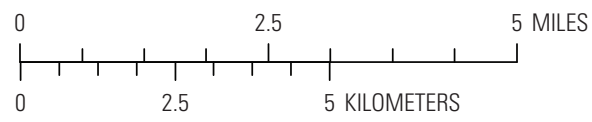

Figure 1. Marsh soil and water-quality sampling sites in the Cache Slough region of the Sacramento-San Joaquin Delta. 
For both parameters, the one-way ANOVA was significant ( $p$ less than 0.0001), and the post hoc analyses indicated that shoot length and stem diameter of $S$. californicus plants were less at the LICB than at the other three sites (figs. 2, 3). In addition, vegetation at the LICB was chlorotic (of a yellow color) compared to the green (healthy) vegetation at the other nearby sites.

\section{Purpose and Scope}

The observations made in 2014 spurred a new study that is described in this report to determine the cause for the stunted and chlorotic vegetation at the LICB. Although we considered many possibilities, this report explains the stunted and chlorotic vegetation at LICB in relation to three potential causes: (1) improper grading of the marsh plain, (2) contamination by pesticides applied to agricultural and urban areas upstream from the site, and (3) nitrogen-deficient soil. We focused on these potential causes for the following reasons. We chose improper grading because, during flood tide, the marsh plain was not flooded to the same depth as in nearby marshes, indicating that the LICB is likely to have a different hydroperiod (the frequency and extent of tidal inundation during a season or longer) compared with that of nearby marshes. Hydroperiod is the chief variable determining the elevation suitable for plant growth in a tidal marsh (Mitsch and Gosselink, 2000). We considered the effect of herbicides on marsh vegetation because of the intensity of pesticide use upstream from the LICB (California Department of Pesticide Regulation, 2015). Lastly, we considered the nitrogen content of the soil, because chlorotic marsh vegetation could be a sign of nitrogen deficiency (Pilbeam, 2015). The study used soil and water samples collected in 2014 or 2015 from four marshes and analyzed for carbon and nitrogen in soil and for pesticides in water. Topographic data for the LICB and a nearby marsh also were measured.

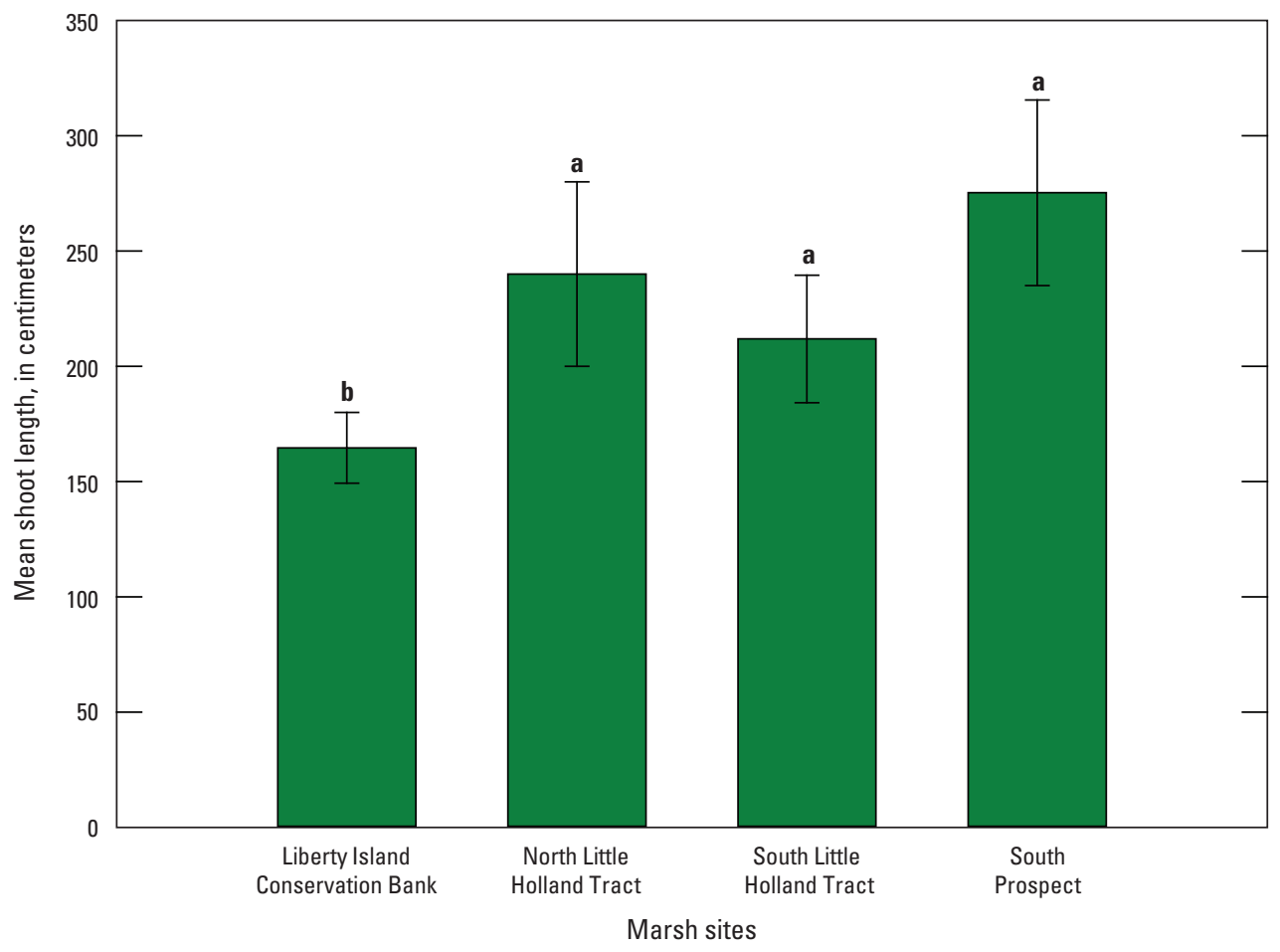

Figure 2. Mean shoot length of California bulrush (Schoenoplectus californicus) collected from four marsh sites in the Cache Slough region of the Sacramento-San Joaquin Delta during September 2014. Letters indicate significant differences among sites from a oneway analysis of variance (ANOVA) and post hoc pairwise comparisons by the Bonferroni method ( $p$ less than 0.0001 for the one-way ANOVA and post hoc comparisons. Vertical bars represent plus and minus one standard deviation error). 


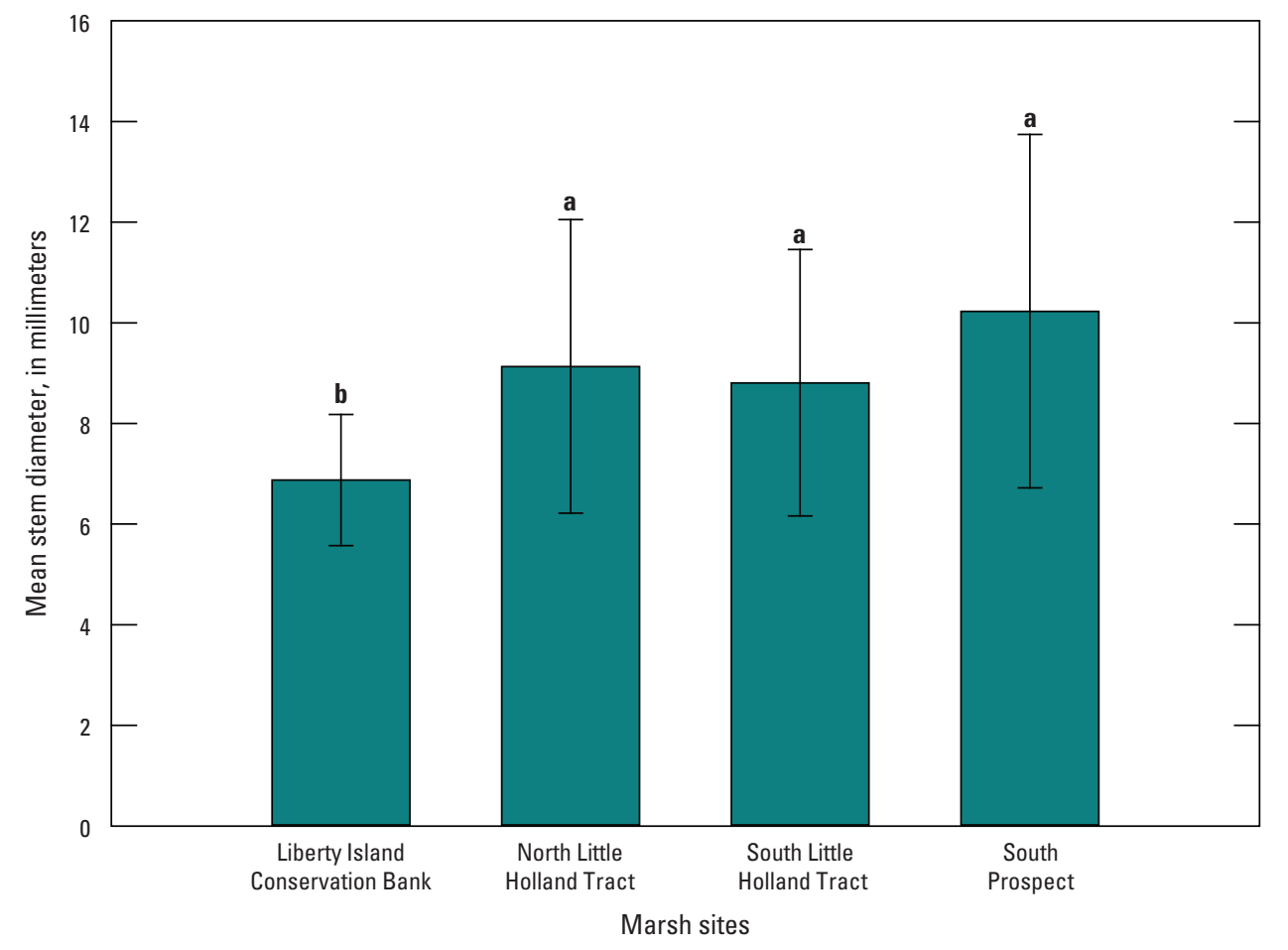

Figure 3. Mean stem diameter of California bulrush collected from four marsh sites in the Cache Slough region of the Sacramento-San Joaquin Delta during September 2014. Letters indicate significant differences among sites from a one-way analysis of variance (ANOVA) and post hoc pairwise comparisons by the Bonferroni method (p less than 0.0001 for the one-way ANOVA and post hoc comparisons. Vertical bars represent plus and minus one standard deviation error).

\section{Field and Laboratory Methods}

The approach for this study included three parts: (1) an elevation survey of the marsh plain in the LICB and north Little Holland Tract (the closest marsh in the most similar hydrodynamic setting in northern Cache Slough region), (2) an analysis of carbon and nitrogen in soil samples collected in each of the four wetland sites in September 2014, and (3) water sampling and analysis for pesticides during the summer of 2015 .

\section{Elevation Survey}

An elevation survey was carried out in November 2015 using RTK-GPS (real-time kinematic global positioning system) in north Little Holland Tract and the LICB. A Trimble R7 GNSS receiver and Zephyr model 2 antenna on a 2-meter, fixed-height tripod were used to collect static data (base station), and a Trimble R8 GNSS model 3 receiver on a 2-meter, fixed-height, carbon-fiber rod (rover unit) was used to collect location data at the marsh sites. A Trimble TDL450H was used for radio communication between the base station and rover units, and data were collected using a Trimble TSC3 hand-held unit. Location and elevation measurements were collected at approximately 3 -meter intervals on multiple transects at two marsh sites (north Little Holland Tract and the LICB). Each transect extended from the marsh and waterway interface to the marsh plain, and each transect varied in length. At north Little Holland Tract, 4 transect lines were sampled for a total of 41 measurement points, whereas at the LICB, 5 transect lines were sampled for a total of 72 measurement points.

\section{Soils}

The top 4 centimeters $(\mathrm{cm})$ of soil were collected in 9-cm-diameter, aluminum sample containers in three locations in each of the four sites (LICB, north Little Holland Tract, south Little Holland Tract, and south Prospect marshes; table 1) during September 2014. The sample container was pushed $4 \mathrm{~cm}$ into the soil surface, and a metal spatula was used to dig into the soil and cut away the bottom of the sample from the underlying soil. Soil samples were sealed in their containers and transported on wet ice to the U.S. Geological Survey (USGS) laboratory in Sacramento, California, where they were stored at 3 degrees Celsius $\left({ }^{\circ} \mathrm{C}\right)$. Soil samples were then dried in ovens at $60^{\circ} \mathrm{C}$, weighed, and subsequently ground to 2 millimeters.

Total percentages, by weight, of carbon, organic carbon, nitrogen, and organic nitrogen were determined using a Perkin Elmer CHNS/O elemental analyzer (Perkin Elmer Corporation, Waltham, Mass.), which was calibrated with blanks and acetanilide standards before use, according to a modified version of U.S. Environmental Protection Agency Method 440.0 (Zimmerman and others, 1997). 
Table 1. Surface-water and soil sampling sites in the Cache Slough region of the Sacramento-San Joaquin Delta, California.

[dd, decimal degrees; NA, not applicable; NAD 83, North American Datum of 1983; USGS, U.S. Geological Survey]

\begin{tabular}{|c|c|c|c|c|c|c|c|}
\hline USGS station number & USGS station name & Marsh & Marsh type & $\begin{array}{l}\text { Sample types } \\
\text { collected }\end{array}$ & $\begin{array}{l}\text { Latitude } \\
\text { (dd) }\end{array}$ & $\begin{array}{l}\text { Longitude } \\
\text { (dd) }\end{array}$ & $\begin{array}{c}\text { Horizontal } \\
\text { datum }\end{array}$ \\
\hline 381719121432301 & $\begin{array}{l}\text { Cache Slough at Hass Slough } \\
\text { near Liberty Farms, CA }\end{array}$ & NA & NA & Surface water & 38.28838 & -121.72302 & NAD 83 \\
\hline 382006121401601 & $\begin{array}{l}\text { Liberty Island at Liberty Cut near } \\
\text { Courtland, CA }\end{array}$ & $\begin{array}{l}\text { Liberty Island Conservation Bank } \\
\text { (LICB) }\end{array}$ & $\begin{array}{l}\text { Restored } \\
\text { marsh }\end{array}$ & Surface water & 38.33501 & -121.67111 & NAD 83 \\
\hline 382006121401701 & $\begin{array}{l}\text { Liberty Island Marsh Site } 1 \text { near } \\
\text { Courtland, CA }\end{array}$ & $\begin{array}{l}\text { Liberty Island Conservation Bank } \\
\text { (LICB) }\end{array}$ & $\begin{array}{l}\text { Restored } \\
\text { marsh }\end{array}$ & Soil & 38.33487 & -121.67148 & NAD 83 \\
\hline 381955121401301 & $\begin{array}{l}\text { Liberty Island Marsh Site } 2 \text { near } \\
\text { Courtland, CA }\end{array}$ & $\begin{array}{l}\text { Liberty Island Conservation Bank } \\
\text { (LICB) }\end{array}$ & $\begin{array}{l}\text { Restored } \\
\text { marsh }\end{array}$ & Soil & 38.33203 & -121.67025 & NAD 83 \\
\hline 382006121401602 & $\begin{array}{l}\text { Liberty Island Marsh Site } 3 \text { near } \\
\text { Courtland, CA }\end{array}$ & $\begin{array}{l}\text { Liberty Island Conservation Bank } \\
\text { (LICB) }\end{array}$ & $\begin{array}{l}\text { Restored } \\
\text { marsh }\end{array}$ & Soil & 38.33502 & -121.67123 & NAD 83 \\
\hline 382005121392801 & $\begin{array}{l}\text { Little Holland Tract near } \\
\text { Courtland, CA }\end{array}$ & North Little Holland Tract & $\begin{array}{c}\text { Remnant } \\
\text { natural marsh }\end{array}$ & Surface water & 38.33486 & -121.65780 & NAD 83 \\
\hline 382005121392901 & $\begin{array}{l}\text { Little Holland Tract Marsh Site } 1 \\
\text { near Courtland, CA }\end{array}$ & North Little Holland Tract & $\begin{array}{c}\text { Remnant } \\
\text { natural marsh }\end{array}$ & Soil & 38.33478 & -121.65813 & NAD 83 \\
\hline 382004121392801 & $\begin{array}{l}\text { Little Holland Tract Marsh Site } 2 \\
\text { near Courtland, CA }\end{array}$ & North Little Holland Tract & $\begin{array}{c}\text { Remnant } \\
\text { natural marsh }\end{array}$ & Soil & 38.33443 & -121.65780 & NAD 83 \\
\hline 382007121392301 & $\begin{array}{l}\text { Little Holland Tract Marsh Site } 3 \\
\text { near Courtland, CA }\end{array}$ & North Little Holland Tract & $\begin{array}{c}\text { Remnant } \\
\text { natural marsh }\end{array}$ & Soil & 38.33532 & -121.65625 & NAD 83 \\
\hline 381721121395301 & $\begin{array}{l}\text { Prospect Slough near Liberty } \\
\text { Farms, CA }\end{array}$ & South Little Holland Tract & $\begin{array}{c}\text { Remnant } \\
\text { natural marsh }\end{array}$ & Surface water & 38.28920 & -121.66483 & NAD 83 \\
\hline 381721121394701 & $\begin{array}{l}\text { Prospect Slough Marsh Site } 4 \\
\text { near Liberty Farms, CA }\end{array}$ & South Little Holland Tract & $\begin{array}{c}\text { Remnant } \\
\text { natural marsh }\end{array}$ & Soil & 38.28915 & -121.66307 & NAD 83 \\
\hline 381718121395301 & $\begin{array}{l}\text { Prospect Slough Marsh Site } 5 \\
\text { near Liberty Farms, CA }\end{array}$ & South Little Holland Tract & $\begin{array}{c}\text { Remnant } \\
\text { natural marsh }\end{array}$ & Soil & 38.28833 & -121.66477 & NAD 83 \\
\hline 381718121395201 & $\begin{array}{l}\text { Prospect Slough Marsh Site } 6 \\
\text { near Liberty Farms, CA }\end{array}$ & South Little Holland Tract & $\begin{array}{c}\text { Remnant } \\
\text { natural marsh }\end{array}$ & Soil & 38.28845 & -121.66445 & NAD 83 \\
\hline 381418121405301 & $\begin{array}{l}\text { Prospect Slough at Cache Slough } \\
\text { near Rio Vista, CA }\end{array}$ & South Prospect & $\begin{array}{c}\text { Remnant } \\
\text { natural marsh }\end{array}$ & Surface water & 38.23829 & -121.68128 & NAD 83 \\
\hline 381414121403901 & $\begin{array}{l}\text { Prospect Slough Marsh Site } 1 \\
\text { near Rio Vista, CA }\end{array}$ & South Prospect & $\begin{array}{c}\text { Remnant } \\
\text { natural marsh }\end{array}$ & Soil & 38.23730 & -121.67763 & NAD 83 \\
\hline 381416121403601 & $\begin{array}{l}\text { Prospect Slough Marsh Site } 2 \\
\text { near Rio Vista, CA }\end{array}$ & South Prospect & $\begin{array}{l}\text { Remnant } \\
\text { natural marsh }\end{array}$ & Soil & 38.23788 & -121.67658 & NAD 83 \\
\hline 381420121403501 & $\begin{array}{l}\text { Prospect Slough Marsh Site } 3 \\
\text { near Rio Vista, CA }\end{array}$ & South Prospect & $\begin{array}{c}\text { Remnant } \\
\text { natural marsh }\end{array}$ & Soil & 38.23897 & -121.67643 & NAD 83 \\
\hline
\end{tabular}




\section{Pesticides}

Water samples were collected at one site in each marsh (table 1) and in Cache Slough downstream from Ulatis Creek, a known source of pesticides to the region (Orlando and others, 2004; Weston and Lydy, 2010), in June, July, and August of 2015. Sites were accessed, and samples were collected, by boat on the ebb tide at each site. Samples were collected by submerging 1-liter (L), baked, amber-glass bottles 0.1 meter below the water surface. Two 1-L bottles were collected at each site. Additional bottles were collected for field, quality-control (QC) samples during the study (one each of a trip blank, replicate, matrix spike, and matrix-spike replicate). After collection, all water samples were chilled on wet ice and delivered the same day to the USGS Organic Chemistry Research Laboratory (OCRL) in Sacramento, California. All water samples were processed at the OCRL laboratory within 24 hours of collection to remove suspended material by filtering through 0.7 -micrometer glass-fiber filters (Grade GF/F, Whatman, Piscataway, New Jersey) into precleaned glass bottles. After filtering, the pre-weighed filter papers and captured suspended sediment were allowed to air dry in a fume hood, then placed in aluminum foil, sealed in zip-lock bags, and stored at $-20{ }^{\circ} \mathrm{C}$ for no longer than 30 days following collection prior to extraction and analysis.

Water samples $(1 \mathrm{~L}$ each) were analyzed for pesticides (table 2) using two published analytical methods. Samples were analyzed for 124 current-use pesticides (CUPs) using a gas chromatograph/mass spectrometer (GC/MS) following the method described in Hladik and others (2008). Water samples were also analyzed for an additional 25 CUPs using liquid chromatography tandem mass spectrometry (LC/MS/MS) following the method described in Hladik and Calhoun (2012). Suspended sediments filtered from the water samples were analyzed for 124 CUPs using GC/MS following the method described in Hladik and McWayne (2012).

Field water-quality parameters (temperature, specific conductance, dissolved oxygen concentration, and $\mathrm{pH}$ ) were measured at the time of sample collection using a YSI 6920V2 multi-parameter meter that was calibrated with appropriate standards immediately prior to sampling (table 3 ). 
Table 2. Method detection limits for pesticides in water measured by the U.S. Geological Survey Organic Chemistry Research Laboratory.

[GC/MS, gas chromatography/mass spectrometry; LC/MS/MS, liquid chromatography/tandem mass spectrometry; ng/L, nanograms per liter; NWIS, National Water Information System]

\begin{tabular}{|c|c|c|c|c|c|c|}
\hline Compound & $\begin{array}{c}\text { NWIS } \\
\text { parameter } \\
\text { code }\end{array}$ & $\begin{array}{l}\text { Pesticide } \\
\text { type }\end{array}$ & $\begin{array}{c}\text { Chemical } \\
\text { class }\end{array}$ & $\begin{array}{c}\text { Method } \\
\text { detection limit } \\
(\mathrm{ng} / \mathrm{L})\end{array}$ & $\begin{array}{c}\text { Analytical } \\
\text { method }\end{array}$ & $\begin{array}{l}\text { Matrices } \\
\text { analyzed }\end{array}$ \\
\hline Acetamiprid & 68302 & Insecticide & Neonicotinoid & 3.3 & $\mathrm{LC} / \mathrm{MS} / \mathrm{MS}$ & Water \\
\hline Acibenzolar-S-methyl & 51849 & Fungicide & Benzothiadiazole & 3.0 & GC/MS & Water and sediment \\
\hline Alachlor & 65064 & Herbicide & Chloroacetanilide & 1.7 & $\mathrm{GC} / \mathrm{MS}$ & Water and sediment \\
\hline Allethrin & 66586 & Insecticide & Pyrethroid & 6.0 & GC/MS & Water and sediment \\
\hline Atrazine & 65065 & Herbicide & Triazine & 2.3 & GC/MS & Water and sediment \\
\hline Azoxystrobin & 66589 & Fungicide & Strobilurin & 3.1 & GC/MS & Water and sediment \\
\hline Benefluralin & 51643 & Herbicide & Dinitroaniline & 2.0 & GC/MS & Water and sediment \\
\hline Bifenthrin & 65067 & Insecticide & Pyrethroid & 4.7 & GC/MS & Water and sediment \\
\hline Boscalid & 67550 & Fungicide & Pyridine & 2.8 & GC/MS & Water and sediment \\
\hline Butralin & 68545 & Herbicide & Dinitroaniline & 2.6 & $\mathrm{GC} / \mathrm{MS}$ & Water and sediment \\
\hline Butylate & 65068 & Herbicide & Thiocarbamate & 1.8 & GC/MS & Water and sediment \\
\hline Captan & 68322 & Fungicide & Phthalimide & 10.2 & $\mathrm{GC} / \mathrm{MS}$ & Water and sediment \\
\hline Carbaryl & 65069 & Insecticide & Carbamate & 6.5 & $\mathrm{GC} / \mathrm{MS}$ & Water and sediment \\
\hline Carbendazim & 68548 & Fungicide & Benzimidazole & 4.2 & $\mathrm{LC} / \mathrm{MS} / \mathrm{MS}$ & Water \\
\hline Carbofuran & 65070 & Insecticide & Carbamate & 3.1 & $\mathrm{GC} / \mathrm{MS}$ & Water and sediment \\
\hline Chlorantraniliprole & 51856 & Insecticide & Anthranilic diamide & 4.0 & $\mathrm{LC} / \mathrm{MS} / \mathrm{MS}$ & Water \\
\hline Chlorothalonil & 65071 & Fungicide & Chloronitrile & 4.1 & GC/MS & Water and sediment \\
\hline Chlorpyrifos & 65072 & Insecticide & Organophosphate & 2.1 & GC/MS & Water and sediment \\
\hline Chlorpyrifos oxon & 68216 & Degradate & Organophosphate & 5.0 & GC/MS & Water and sediment \\
\hline Clomazone & 67562 & Herbicide & Isoxazlidinone & 2.5 & GC/MS & Water and sediment \\
\hline Clothianidin & 68221 & Insecticide & Neonicotinoid & 3.9 & LC/MS/MS & Water \\
\hline Coumaphos & 51836 & Insecticide & Organophosphate & 3.1 & GC/MS & Water and sediment \\
\hline Cyantraniliprole & 51862 & Insecticide & Anthranilic diamide & 4.2 & $\mathrm{LC} / \mathrm{MS} / \mathrm{MS}$ & Water \\
\hline Cyazofamid & 51853 & Fungicide & Azole & 4.1 & $\mathrm{LC} / \mathrm{MS} / \mathrm{MS}$ & Water \\
\hline Cycloate & 65073 & Herbicide & Thiocarbamate & 1.1 & GC/MS & Water and sediment \\
\hline Cyfluthrin & 65074 & Insecticide & Pyrethroid & 5.2 & GC/MS & Water and sediment \\
\hline Cyhalofop-butyl & 68360 & Herbicide & Aryloxyphenoxypropionate & 1.9 & GC/MS & Water and sediment \\
\hline Cyhalothrin & 68354 & Insecticide & Pyrethroid & 2.0 & GC/MS & Water and sediment \\
\hline Cymoxanil & 51861 & Fungicide & Unclassified & 3.9 & $\mathrm{LC} / \mathrm{MS} / \mathrm{MS}$ & Water \\
\hline Cypermethrin & 65075 & Insecticide & Pyrethroid & 5.6 & GC/MS & Water and sediment \\
\hline
\end{tabular}


Table 2. Method detection limits for pesticides in water measured by the U.S. Geological Survey Organic Chemistry Research Laboratory.-Continued

[GC/MS, gas chromatography/mass spectrometry; LC/MS/MS, liquid chromatography/tandem mass spectrometry; ng/L, nanograms per liter; NWIS, National Water Information System]

\begin{tabular}{|c|c|c|c|c|c|c|}
\hline Compound & $\begin{array}{c}\text { NWIS } \\
\text { parameter } \\
\text { code }\end{array}$ & $\begin{array}{l}\text { Pesticide } \\
\text { type }\end{array}$ & $\begin{array}{l}\text { Chemical } \\
\text { class }\end{array}$ & $\begin{array}{c}\text { Method } \\
\text { detection limit } \\
\text { (ng/L) }\end{array}$ & $\begin{array}{c}\text { Analytical } \\
\text { method }\end{array}$ & $\begin{array}{l}\text { Matrices } \\
\text { analyzed }\end{array}$ \\
\hline Cyproconazole & 66593 & Fungicide & Triazole & 4.7 & GC/MS & Water and sediment \\
\hline Cyprodinil & 67574 & Fungicide & Pyrimidine & 7.4 & GC/MS & Water and sediment \\
\hline DCPA & 65076 & Herbicide & Benzenedicarboxylic acid & 2.0 & $\mathrm{GC} / \mathrm{MS}$ & Water and sediment \\
\hline p,p'-DDD & 65094 & Degradate & Organochlorine & 4.1 & $\mathrm{GC} / \mathrm{MS}$ & Water and sediment \\
\hline p,p'-DDE & 65095 & Degradate & Organochlorine & 3.6 & GC/MS & Water and sediment \\
\hline p,p'-DDT & 65096 & Insecticide & Organochlorine & 4.0 & GC/MS & Water and sediment \\
\hline Deltamethrin & 65077 & Insecticide & Pyrethroid & 3.5 & GC/MS & Water and sediment \\
\hline Desthio-Prothioconazole & 51865 & Fungicide & Azole & 3.0 & LC/MS/MS & Water \\
\hline Desulfinylfipronil & 66607 & Degradate & Phenylpyrazole & 1.6 & GC/MS & Water and sediment \\
\hline Desulfinylfipronil amide & 68570 & Degradate & Phenylpyrazole & 3.2 & GC/MS & Water and sediment \\
\hline Diazinon & 65078 & Insecticide & Organophosphate & 0.9 & GC/MS & Water and sediment \\
\hline Diazinon oxon & 68236 & Degradate & Organophosphate & 5.0 & GC/MS & Water and sediment \\
\hline 3,4-Dichloroaniline (3,4-DCA) & 66584 & Degradate & Urea & 3.2 & $\mathrm{LC} / \mathrm{MS} / \mathrm{MS}$ & Water \\
\hline 3,5-Dichloroaniline (3,5-DCA) & 67536 & Degradate & Aniline & 7.6 & GC/MS & Water and sediment \\
\hline 3,4-Dichlorophenylurea (DCPU) & 68226 & Degradate & Urea & 3.4 & $\mathrm{LC} / \mathrm{MS} / \mathrm{MS}$ & Water \\
\hline Difenoconazole & 67582 & Fungicide & Triazole & 10.5 & GC/MS & Water and sediment \\
\hline (E)-Dimethomorph & 67587 & Fungicide & Morpholine & 6.0 & GC/MS & Water and sediment \\
\hline Dinotefuran & 68379 & Insecticide & Neonicotinoid & 4.5 & LC/MS/MS & Water \\
\hline Dithiopyr & 51837 & Herbicide & Pyridine & 1.6 & GC/MS & Water and sediment \\
\hline Diuron & 66598 & Herbicide & Urea & 3.2 & LC/MS/MS & Water \\
\hline EPTC & 65080 & Herbicide & Thiocarbamate & 1.5 & GC/MS & Water and sediment \\
\hline Esfenvalerate & 65081 & Insecticide & Pyrethroid & 3.9 & GC/MS & Water and sediment \\
\hline Ethaboxam & 51855 & Fungicide & Unclassified & 3.8 & $\mathrm{LC} / \mathrm{MS} / \mathrm{MS}$ & Water \\
\hline Ethalfluralin & 65082 & Herbicide & Aniline & 3.0 & GC/MS & Water and sediment \\
\hline Etofenprox & 67604 & Insecticide & Pyrethroid & 2.2 & GC/MS & Water and sediment \\
\hline Famoxadone & 67609 & Fungicide & Oxazole & 2.5 & GC/MS & Water and sediment \\
\hline Fenamidone & 51848 & Fungicide & Imidazole & 5.1 & GC/MS & Water and sediment \\
\hline Fenarimol & 67613 & Fungicide & Pyrimidine & 6.5 & GC/MS & Water and sediment \\
\hline Fenbuconazole & 67618 & Fungicide & Triazole & 5.2 & GC/MS & Water and sediment \\
\hline Fenhexamide & 67622 & Fungicide & Anilide & 7.6 & GC/MS & Water and sediment \\
\hline Fenpropathrin & 65083 & Insecticide & Pyrethroid & 4.1 & GC/MS & Water and sediment \\
\hline
\end{tabular}


Table 2. Method detection limits for pesticides in water measured by the U.S. Geological Survey Organic Chemistry Research Laboratory.-Continued

[GC/MS, gas chromatography/mass spectrometry; LC/MS/MS, liquid chromatography/tandem mass spectrometry; ng/L, nanograms per liter; NWIS, National Water Information System]

\begin{tabular}{|c|c|c|c|c|c|c|}
\hline Compound & $\begin{array}{c}\text { NWIS } \\
\text { parameter } \\
\text { code }\end{array}$ & $\begin{array}{l}\text { Pesticide } \\
\text { type }\end{array}$ & $\begin{array}{l}\text { Chemical } \\
\text { class }\end{array}$ & $\begin{array}{c}\text { Method } \\
\text { detection limit } \\
\text { (ng/L) }\end{array}$ & $\begin{array}{c}\text { Analytical } \\
\text { method }\end{array}$ & $\begin{array}{l}\text { Matrices } \\
\text { analyzed }\end{array}$ \\
\hline Fenpyroximate & 51838 & Insecticide & Pyrazole & 5.2 & GC/MS & Water and sediment \\
\hline Fenthion & 51839 & Insecticide & Organophosphate & 5.5 & GC/MS & Water and sediment \\
\hline Fipronil & 66604 & Insecticide & Phenylpyrazole & 2.9 & $\mathrm{GC} / \mathrm{MS}$ & Water and sediment \\
\hline Fipronil sulfide & 66610 & Degradate & Phenylpyrazole & 1.8 & GC/MS & Water and sediment \\
\hline Fipronil sulfone & 66613 & Degradate & Phenylpyrazole & 3.5 & GC/MS & Water and sediment \\
\hline Fludioxinil & 67640 & Fungicide & Pyrrole & 7.3 & GC/MS & Water and sediment \\
\hline Flufenacet & 51840 & Herbicide & Anilide & 4.7 & GC/MS & Water and sediment \\
\hline Flumetralin & 51841 & Plant growth regulator & Dinitroaniline & 5.8 & GC/MS & Water and sediment \\
\hline Fluopicolide & 51852 & Fungicide & Pyrimidine & 3.9 & $\mathrm{GC} / \mathrm{MS}$ & Water and sediment \\
\hline Fluoxastrobin & 67645 & Fungicide & Strobilurin & 9.5 & GC/MS & Water and sediment \\
\hline Fluridone & 51864 & Herbicide & Unclassified & 3.7 & $\mathrm{LC} / \mathrm{MS} / \mathrm{MS}$ & Water \\
\hline Fluxapyroxad & 51851 & Fungicide & Anilide & 4.8 & GC/MS & Water and sediment \\
\hline Hexazinone & 65085 & Herbicide & Triazone & 8.4 & GC/MS & Water and sediment \\
\hline Imazalil & 67662 & Fungicide & Triazole & 10.5 & GC/MS & Water and sediment \\
\hline Imidacloprid & 68426 & Insecticide & Neonicotinoid & 3.8 & $\mathrm{LC} / \mathrm{MS} / \mathrm{MS}$ & Water \\
\hline Indoxacarb & 68898 & Insecticide & Oxadiazine & 4.9 & GC/MS & Water and sediment \\
\hline Iprodione & 66617 & Fungicide & Dicarboxamide & 4.4 & GC/MS & Water and sediment \\
\hline Kresoxim-methyl & 67670 & Fungicide & Strobilurin & 4.0 & $\mathrm{GC} / \mathrm{MS}$ & Water and sediment \\
\hline Malathion & 65087 & Insecticide & Organophosphate & 3.7 & GC/MS & Water and sediment \\
\hline Malathion oxon & 68240 & Degradate & Organophosphate & 5.0 & GC/MS & Water and sediment \\
\hline Mandipropamid & 51854 & Fungicide & Mandelamide & 3.3 & LC/MS/MS & Water \\
\hline Metalaxyl & 68437 & Fungicide & Phenylamide & 5.1 & GC/MS & Water and sediment \\
\hline Metconazole & 66620 & Fungicide & Azole & 5.2 & GC/MS & Water and sediment \\
\hline Methidathion & 65088 & Insecticide & Organophosphate & 7.2 & GC/MS & Water and sediment \\
\hline Methoprene & 66623 & Insecticide & Terpene & 6.4 & GC/MS & Water and sediment \\
\hline
\end{tabular}


Table 2. Method detection limits for pesticides in water measured by the U.S. Geological Survey Organic Chemistry Research Laboratory.-Continued

[GC/MS, gas chromatography/mass spectrometry; LC/MS/MS, liquid chromatography/tandem mass spectrometry; ng/L, nanograms per liter; NWIS, National Water Information System]

\begin{tabular}{|c|c|c|c|c|c|c|}
\hline Compound & $\begin{array}{c}\text { NWIS } \\
\text { parameter } \\
\text { code }\end{array}$ & $\begin{array}{l}\text { Pesticide } \\
\text { type }\end{array}$ & $\begin{array}{l}\text { Chemical } \\
\text { class }\end{array}$ & $\begin{array}{c}\text { Method } \\
\text { detection limit } \\
\text { (ng/L) }\end{array}$ & $\begin{array}{l}\text { Analytical } \\
\text { method }\end{array}$ & $\begin{array}{l}\text { Matrices } \\
\text { analyzed }\end{array}$ \\
\hline Methoxyfenozide & 68647 & Insecticide & Diacylhydrazine & 2.7 & LC/MS/MS & Water \\
\hline Methylparathion & 65089 & Insecticide & Organophosphate & 3.4 & GC/MS & Water and sediment \\
\hline Metolachlor & 65090 & Herbicide & Chloroacetanilide & 1.5 & GC/MS & Water and sediment \\
\hline Molinate & 65091 & Herbicide & Thiocarbamate & 3.2 & $\mathrm{GC} / \mathrm{MS}$ & Water and sediment \\
\hline Myclobutanil & 66632 & Fungicide & Triazole & 6.0 & GC/MS & Water and sediment \\
\hline$N$-(3,4-Dichlorophenyl)- $N$ '-methylurea (DCPMU) & 68231 & Degradate & Urea & 3.5 & $\mathrm{LC} / \mathrm{MS} / \mathrm{MS}$ & Water \\
\hline Napropamide & 65092 & Herbicide & Amide & 8.2 & GC/MS & Water and sediment \\
\hline Novaluron & 68655 & Herbicide & Benzoylurea & 2.9 & GC/MS & Water and sediment \\
\hline Oryzalin & 68663 & Herbicide & 2,6-Dinitroaniline & 5.0 & $\mathrm{LC} / \mathrm{MS} / \mathrm{MS}$ & Water \\
\hline Oxadiazon & 51843 & Herbicide & Oxadiazolone & 2.1 & GC/MS & Water and sediment \\
\hline Oxyfluorfen & 65093 & Herbicide & Nitrophenyl ether & 3.1 & GC/MS & Water and sediment \\
\hline Paclobutrazol & 51846 & Fungicide & Triazole & 6.2 & GC/MS & Water and sediment \\
\hline Pebulate & 65097 & Herbicide & Thiocarbamate & 2.3 & GC/MS & Water and sediment \\
\hline Pendimethalin & 65098 & Herbicide & Aniline & 2.3 & GC/MS & Water and sediment \\
\hline Penoxsulam & 51863 & Herbicide & Triazolopyrimidine & 3.5 & $\mathrm{LC} / \mathrm{MS} / \mathrm{MS}$ & Water \\
\hline Pentachloroanisole (PCA) & 66637 & Insecticide & Organochlorine & 4.7 & GC/MS & Water and sediment \\
\hline Pentachloronitrobenzene (PCNB) & 66639 & Fungicide & Organochlorine & 3.1 & GC/MS & Water and sediment \\
\hline Permethrin & 65099 & Insecticide & Pyrethroid & 3.4 & GC/MS & Water and sediment \\
\hline Phenothrin & 65100 & Insecticide & Pyrethroid & 5.1 & GC/MS & Water and sediment \\
\hline Phosmet & 65101 & Insecticide & Organophosphate & 4.4 & GC/MS & Water and sediment \\
\hline Picoxystrobin & 51850 & Fungicide & Strobilurin & 4.2 & GC/MS & Water and sediment \\
\hline Piperonyl butoxide & 65102 & Synergist & Unclassified & 2.3 & GC/MS & Water and sediment \\
\hline Prodiamine & 51844 & Herbicide & Dinitroaniline & 5.2 & GC/MS & Water and sediment \\
\hline Prometon & 67702 & Herbicide & Triazine & 2.5 & GC/MS & Water and sediment \\
\hline Prometryn & 65103 & Herbicide & Triazine & 1.8 & GC/MS & Water and sediment \\
\hline Propanil & 66641 & Herbicide & Anilide & 10.1 & GC/MS & Water and sediment \\
\hline Propargite & 68677 & Insecticide & Sulfite ester & 6.1 & GC/MS & Water and sediment \\
\hline Propiconazole & 66643 & Fungicide & Azole & 5.0 & GC/MS & Water and sediment \\
\hline Propyzamide & 67706 & Herbicide & Benzamide & 5.0 & GC/MS & Water and sediment \\
\hline Pyraclostrobin & 66646 & Fungicide & Strobilurin & 2.9 & GC/MS & Water and sediment \\
\hline Pyridaben & 68908 & Insecticide & Pyridazinone & 5.4 & GC/MS & Water and sediment \\
\hline
\end{tabular}


Table 2. Method detection limits for pesticides in water measured by the U.S. Geological Survey Organic Chemistry Research Laboratory.-Continued

[GC/MS, gas chromatography/mass spectrometry; LC/MS/MS, liquid chromatography/tandem mass spectrometry; ng/L, nanograms per liter; NWIS, National Water Information System]

\begin{tabular}{|c|c|c|c|c|c|c|}
\hline Compound & $\begin{array}{c}\text { NWIS } \\
\text { parameter } \\
\text { code }\end{array}$ & $\begin{array}{l}\text { Pesticide } \\
\text { type }\end{array}$ & $\begin{array}{l}\text { Chemical } \\
\text { class }\end{array}$ & $\begin{array}{c}\text { Method } \\
\text { detection limit } \\
\text { (ng/L) }\end{array}$ & $\begin{array}{c}\text { Analytical } \\
\text { method }\end{array}$ & $\begin{array}{l}\text { Matrices } \\
\text { analyzed }\end{array}$ \\
\hline Pyrimethanil & 67717 & Fungicide & Pyrmidine & 4.1 & GC/MS & Water and sediment \\
\hline Quinoxyfen & 51847 & Fungicide & Quinoline & 3.3 & GC/MS & Water and sediment \\
\hline Resmethrin & 65104 & Insecticide & Pyrethroid & 5.7 & GC/MS & Water and sediment \\
\hline Simazine & 65105 & Herbicide & Triazine & 5.0 & GC/MS & Water and sediment \\
\hline Tebuconazole & 66649 & Fungicide & Azole & 3.7 & GC/MS & Water and sediment \\
\hline Tefluthrin & 67731 & Insecticide & Pyrethroid & 4.2 & GC/MS & Water and sediment \\
\hline Tetraconazole & 66654 & Fungicide & Azole & 5.6 & GC/MS & Water and sediment \\
\hline Tetradifon & 51651 & Insecticide & Bridged diphenyl & 3.8 & GC/MS & Water and sediment \\
\hline Tetramethrin & 66657 & Insecticide & Pyrethroid & 2.9 & GC/MS & Water and sediment \\
\hline Thiabendazole & 67161 & Fungicide & Benzimidazole & 3.6 & LC/MS/MS & Water \\
\hline Thiacloprid & 68485 & Insecticide & Neonicotinoid & 3.2 & $\mathrm{LC} / \mathrm{MS} / \mathrm{MS}$ & Water \\
\hline Triadimefon & 67741 & Fungicide & Triazole & 8.9 & GC/MS & Water and sediment \\
\hline Triadimenol & 67746 & Fungicide & Triazole & 8.0 & GC/MS & Water and sediment \\
\hline Triallate & 68710 & Herbicide & Carbamate & 2.4 & GC/MS & Water and sediment \\
\hline Tribufos & 68711 & Herbicide & Organophosphate & 3.1 & GC/MS & Water and sediment \\
\hline Trifloxystrobin & 66660 & Fungicide & Strobilurin & 4.7 & GC/MS & Water and sediment \\
\hline Triflumizole & 67753 & Fungicide & Azole & 6.1 & GC/MS & Water and sediment \\
\hline Trifluralin & 65108 & Herbicide & Aniline & 2.1 & GC/MS & Water and sediment \\
\hline Triticonazole & 67758 & Fungicide & Azole & 6.9 & GC/MS & Water and sediment \\
\hline Zoxamide & 67768 & Fungicide & Benzamide & 3.5 & GC/MS & Water and sediment \\
\hline
\end{tabular}


Table 3. Field water-quality parameter data measured at collection sites in 2015 during water-sample collection in the Cache Slough region of the Sacramento-San Joaquin Delta, California.

[Numbers in brackets are U.S. Geological Survey (USGS) National Water Information System (NWIS) parameter codes. Abbreviations: hh:mm, hours:minutes; mg/L, milligrams per liter; NA, data not collected; ${ }^{\circ} \mathrm{C}$, degrees Celsius; $\mu \mathrm{S} / \mathrm{cm}$, microsiemens per centimeter]

\begin{tabular}{|c|c|c|c|c|c|c|c|}
\hline $\begin{array}{c}\text { USGS station } \\
\text { number }\end{array}$ & USGS station name & Short site name & $\begin{array}{l}\text { Sample } \\
\text { time } \\
\text { (hh:mm) }\end{array}$ & $\begin{array}{c}\text { Water } \\
\text { temperature } \\
\left({ }^{\circ} \mathrm{C}\right) \\
{[00010]}\end{array}$ & $\begin{array}{c}\text { Specific } \\
\text { conductance } \\
(\mu \mathrm{S} / \mathrm{cm} \text { at } \\
\left.25^{\circ} \mathrm{C}\right) \\
{[00095]}\end{array}$ & $\begin{array}{c}\text { Dissolved } \\
\text { oxygen } \\
\text { (mg/L) } \\
{[00300]}\end{array}$ & $\begin{array}{c}\mathrm{pH} \\
{[00400]}\end{array}$ \\
\hline \multicolumn{8}{|c|}{ Sample date: June 3, 2015} \\
\hline 381719121432301 & Cache Slough at Hass Slough near Liberty Farms, CA & Ulatis Creek & $10: 45$ & NA & 217 & NA & 7.6 \\
\hline 382006121401601 & Liberty Island at Liberty Cut near Courtland, CA & Liberty Island Conservation Bank (LICB) & $11: 30$ & 20.6 & 350 & NA & 7.3 \\
\hline 382005121392801 & Little Holland Tract near Courtland, CA & North Little Holland Tract & $11: 50$ & 22.1 & 263 & NA & 7.5 \\
\hline 381721121395301 & Prospect Slough near Liberty Farms, CA & South Little Holland Tract & 13:00 & 20.8 & 221 & NA & 8.4 \\
\hline 381418121405301 & Prospect Slough at Cache Slough near Rio Vista, CA & South Prospect & $12: 35$ & 20.0 & 207 & NA & 7.8 \\
\hline \multicolumn{8}{|c|}{ Sample date: July 6, 2015} \\
\hline 381719121432301 & Cache Slough at Hass Slough near Liberty Farms, CA & Ulatis Creek & $10: 40$ & 21.0 & 201 & 9.0 & 8.3 \\
\hline 382006121401601 & Liberty Island at Liberty Cut near Courtland, CA & Liberty Island Conservation Bank (LICB) & $11: 10$ & 21.4 & 255 & 6.8 & 7.6 \\
\hline 382005121392801 & Little Holland Tract near Courtland, CA & North Little Holland Tract & $11: 25$ & 20.1 & 219 & 8.6 & 8.0 \\
\hline 381721121395301 & Prospect Slough near Liberty Farms, CA & South Little Holland Tract & $11: 45$ & 21.2 & 209 & 8.9 & 8.2 \\
\hline 381418121405301 & Prospect Slough at Cache Slough near Rio Vista, CA & South Prospect & $12: 10$ & 22.2 & 217 & 9.0 & 7.9 \\
\hline \multicolumn{8}{|c|}{ Sample date: August 13, 2015} \\
\hline 381719121432301 & Cache Slough at Hass Slough near Liberty Farms, CA & Ulatis Creek & $10: 30$ & 21.4 & 206 & 9.4 & 8.5 \\
\hline 382006121401601 & Liberty Island at Liberty Cut near Courtland, CA & Liberty Island Conservation Bank (LICB) & $11: 00$ & 21.6 & 320 & 6.1 & 7.5 \\
\hline 382005121392801 & Little Holland Tract near Courtland, CA & North Little Holland Tract & $11: 15$ & 22.9 & 253 & 7.7 & 7.9 \\
\hline 381721121395301 & Prospect Slough near Liberty Farms, CA & South Little Holland Tract & $11: 35$ & 21.8 & 207 & 8.5 & 8.1 \\
\hline 381418121405301 & Prospect Slough at Cache Slough near Rio Vista, CA & South Prospect & $11: 52$ & 22.0 & 192 & 8.3 & 8.0 \\
\hline
\end{tabular}




\section{Quality Assurance and Quality Control}

\section{Soil Samples}

Replicates and standards were analyzed every five samples to assess instrument stability. Replicate samples were re-analyzed if the relative percentage difference between the two replicates was greater than 20 percent. The method detection limits were 0.15 percent by weight for carbon and 0.10 percent by weight for nitrogen. Statistical analysis of the organic carbon and nitrogen (percentage by weight) in soils was done with one-way ANOVA and post hoc pairwise comparisons using the Bonferroni method.

\section{Pesticides}

Four quality-control samples (a trip blank, replicate, matrix spike, and matrix-spike replicate) were collected and analyzed. The trip blank consisted of a 1-L amber-glass bottle filled with pesticide-grade blank water, which was taken into the field (chilled on ice) during the August sampling and opened to the atmosphere while water samples were collected at one field site. The trip blank was processed in the same manner as the environmental samples and was then analyzed by GC/MS. There were no pesticides detected in the trip blank.

One replicate sample was collected during the June 2015 sampling (Liberty Island at Liberty Cut near Courtland, Calif.) and analyzed by GC/MS. Eight pesticides were detected in the replicate sample as well as the corresponding environmental sample. The relative standard deviations of the paired detections ranged from 1 to 7 percent, which met the data-quality objective of less than 25 percent. There were no unpaired detections of any pesticides either in the environmental or replicate sample.

One matrix-spike sample and one matrix-spike replicate sample were collected during the July sampling (Prospect Slough near Liberty Farms, Calif.) and analyzed by LC/ MS/MS. Both samples were spiked with the full range of analytical compounds (table 2). Recoveries for all compounds ranged from 72 to 119 percent, which met the data-quality objective of 70-130 percent. The relative standard deviations between the matrix spike and matrix-spike replicate samples for all compounds ranged from 2 to 6 percent, which met the data quality objective of less than 25 percent. In addition to the four QC samples described previously, analyte recovery was assessed in all samples by the addition of surrogate compounds in the laboratory. Recoveries for all surrogate compounds met the data-quality objective of 70-130 percent.

\section{Results}

\section{Elevation Survey}

Elevation data for north Little Holland Tract and the LICB are shown in tables 4 and 5, respectively, and in figure 4 , which shows the elevation of survey points at both sites. The mean elevation of the marsh plain was determined to be 1.42 meters relative to the North American Vertical Datum of 1988 (NAVD 88) at north Little Holland Tract, whereas at the LICB, the mean elevation of the marsh plan was 1.68 meters relative to the NAVD 88. Marsh elevations could not be directly compared to mean tide elevation because the closest reliable tidal benchmark is at Port Chicago, Calif., which is more than 43 kilometers from the sites. This distance is too great for conversion of the NAVD 88 elevations to tidal elevations without unacceptable error. Regardless, the mean difference of $26 \mathrm{~cm}$ between the elevations at the LICB and north Little Holland Tract represents 25 percent of the 1-meter habitable range of elevation for marsh vegetation in the delta region (Swanson and others, 2015). This result indicates that the elevation of the LICB is too high for the formation of a fully functional micro-tidal marsh in this region of the delta.

\section{Soil Samples}

The results of the soil analyses showed that there were no statistically significant differences among the four sites for total carbon, organic carbon, total nitrogen, or total organic nitrogen in the top $4 \mathrm{~cm}$ of the soil (table 6; fig. 5). This indicates that soil nitrogen in the surface of the marshes is not likely to be limiting plant growth at the LICB. Because we did not sample deeper in the soil, however, we cannot be sure that nitrogen, or some other nutrient, is not lacking at a greater depth in the soils at the LICB. We also did not measure bulk density, which could have shown effects from compaction following restoration at the LICB compared with the other marsh sites, which were not disturbed by heavy machinery (Sloey and Hester, 2016). 


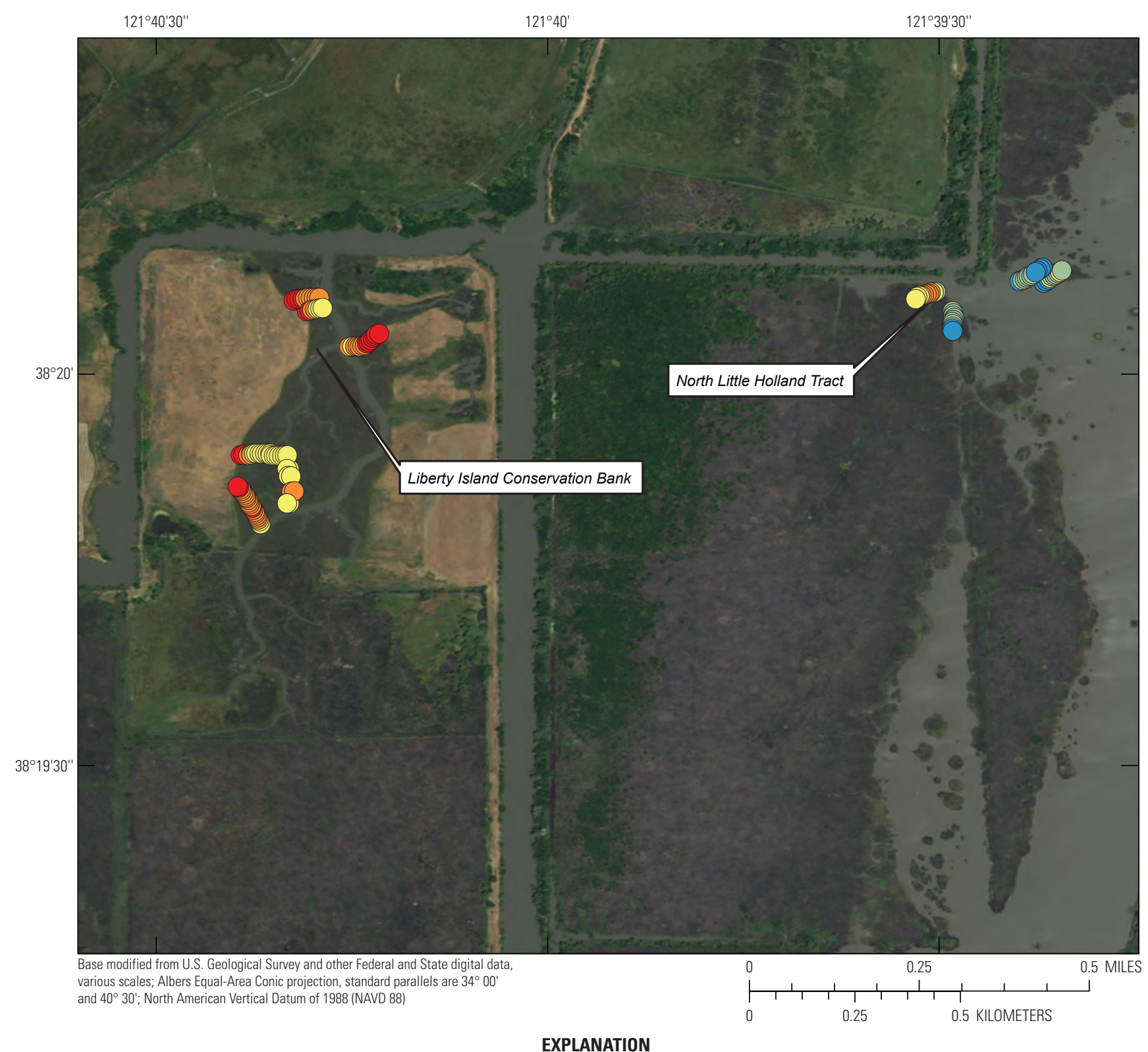

Global Positioning System survey points, elevation in meters
1.02 to 1.31
$>1.31$ to 1.49
$>>1.49$ to 1.63
$>1.63$ to 1.72
$>1.72$ to 1.86

Figure 4. The RTK-GPS (real-time kinematic global positioning system) survey-point elevations, relative to the North American Vertical Datum of 1988, for two marsh sites in the Cache Slough region of the Sacramento-San Joaquin Delta, November 2015. 
Table 4. Elevation and coordinate data from the Global Positioning System survey at the north Little Holland Tract marsh site, November 2015.

[Horizontal coordinates are reported using the North American Datum of 1983; NAD 83. Elevation data is reported in meters (m) referenced to the North American Vertical Datum of 1988; NAVD 88. Abbreviation: dd, decimal degrees]

\begin{tabular}{|c|c|c|c|c|c|c|c|c|c|}
\hline $\begin{array}{c}\text { Site } \\
\text { number }\end{array}$ & Transect & $\begin{array}{l}\text { Latitude } \\
\text { (dd) }\end{array}$ & $\begin{array}{l}\text { Longitude } \\
\text { (dd) }\end{array}$ & $\begin{array}{c}\text { Elevation } \\
(\mathrm{m})\end{array}$ & $\begin{array}{c}\text { Site } \\
\text { number }\end{array}$ & Transect & $\begin{array}{l}\text { Latitude } \\
\text { (dd) }\end{array}$ & $\begin{array}{l}\text { Longitude } \\
\text { (dd) }\end{array}$ & $\begin{array}{c}\text { Elevation } \\
\text { (m) }\end{array}$ \\
\hline 1 & 1 & 38.33509 & -121.65840 & 1.49 & 21 & 3 & 38.33531 & -121.65662 & 1.23 \\
\hline 3 & 1 & 38.33506 & -121.65851 & 1.65 & 23 & 3 & 38.33536 & -121.65650 & 1.46 \\
\hline 4 & 1 & 38.33504 & -121.65855 & 1.66 & 24 & 3 & 38.33539 & -121.65646 & 1.41 \\
\hline 7 & 1 & 38.33500 & -121.65870 & 1.71 & 27 & 3 & 38.33546 & -121.65633 & 1.40 \\
\hline 8 & 1 & 38.33499 & -121.65875 & 1.62 & 28 & 3 & 38.33550 & -121.65627 & 1.29 \\
\hline 9 & 1 & 38.33497 & -121.65879 & 1.62 & 29 & 3 & 38.33553 & -121.65623 & 1.22 \\
\hline 10 & 1 & 38.33493 & -121.65883 & 1.58 & 30 & 3 & 38.33557 & -121.65617 & 1.25 \\
\hline 14 & 2 & 38.33453 & -121.65801 & 1.34 & 34 & 4 & 38.33534 & -121.65600 & 1.29 \\
\hline 15 & 2 & 38.33448 & -121.65803 & 1.34 & 35 & 4 & 38.33538 & -121.65596 & 1.51 \\
\hline 16 & 2 & 38.33444 & -121.65804 & 1.37 & 36 & 4 & 38.33541 & -121.65593 & 1.43 \\
\hline 17 & 2 & 38.33439 & -121.65804 & 1.39 & 37 & 4 & 38.33543 & -121.65588 & 1.45 \\
\hline 18 & 2 & 38.33434 & -121.65804 & 1.34 & 38 & 4 & 38.33545 & -121.65584 & 1.48 \\
\hline 19 & 2 & 38.33430 & -121.65805 & 1.31 & 39 & 4 & 38.33548 & -121.65580 & 1.47 \\
\hline \multirow[t]{2}{*}{20} & 2 & 38.33425 & -121.65804 & 1.29 & 40 & 4 & 38.33551 & -121.65576 & 1.54 \\
\hline & & & & & 41 & 4 & 38.33554 & -121.65572 & 1.39 \\
\hline
\end{tabular}


Table 5. Elevation and coordinate data from the Global Positioning System survey at the Liberty Island Conservation Bank marsh site, November 2015.

[Horizontal coordinates are reported using the North American Datum of 1983; NAD 83. Elevation data is reported in meters (m) referenced to the North American Vertical Datum of 1988; NAVD 88. Abbreviation: dd, decimal degrees]

\begin{tabular}{|c|c|c|c|c|c|c|c|c|c|}
\hline $\begin{array}{c}\text { Site } \\
\text { number }\end{array}$ & Transect & $\begin{array}{l}\text { Latitude } \\
\text { (dd) }\end{array}$ & $\begin{array}{l}\text { Longitude } \\
\text { (dd) }\end{array}$ & $\begin{array}{l}\text { Elevation } \\
\text { (m) }\end{array}$ & $\begin{array}{c}\text { Site } \\
\text { number }\end{array}$ & Transect & $\begin{array}{l}\text { Latitude } \\
\text { (dd) }\end{array}$ & $\begin{array}{l}\text { Longitude } \\
\text { (dd) }\end{array}$ & $\begin{array}{l}\text { Elevation } \\
\text { (m) }\end{array}$ \\
\hline 1 & 1 & 38.33495 & -121.67153 & 1.69 & 37 & 3 & 38.33091 & -121.67324 & 1.75 \\
\hline 2 & 1 & 38.33495 & -121.67159 & 1.69 & 38 & 3 & 38.33094 & -121.67327 & 1.76 \\
\hline 3 & 1 & 38.33495 & -121.67165 & 1.66 & 39 & 4 & 38.33161 & -121.67315 & 1.79 \\
\hline 4 & 1 & 38.33495 & -121.67171 & 1.69 & 40 & 4 & 38.33161 & -121.67310 & 1.74 \\
\hline 5 & 1 & 38.33495 & -121.67177 & 1.69 & 41 & 4 & 38.33161 & -121.67304 & 1.71 \\
\hline 6 & 1 & 38.33494 & -121.67183 & 1.69 & 42 & 4 & 38.33162 & -121.67298 & 1.70 \\
\hline 7 & 1 & 38.33493 & -121.67189 & 1.76 & 43 & 4 & 38.33163 & -121.67292 & 1.69 \\
\hline 8 & 1 & 38.33493 & -121.67196 & 1.74 & 44 & 4 & 38.33164 & -121.67286 & 1.62 \\
\hline 9 & 1 & 38.33492 & -121.67201 & 1.78 & 45 & 4 & 38.33164 & -121.67281 & 1.63 \\
\hline 10 & 1 & 38.33490 & -121.67208 & 1.76 & 46 & 4 & 38.33164 & -121.67274 & 1.61 \\
\hline 11 & 2 & 38.33467 & -121.67179 & 1.79 & 47 & 4 & 38.33163 & -121.67269 & 1.61 \\
\hline 12 & 2 & 38.33468 & -121.67174 & 1.74 & 48 & 4 & 38.33164 & -121.67262 & 1.61 \\
\hline 13 & 2 & 38.33470 & -121.67168 & 1.71 & 49 & 4 & 38.33163 & -121.67257 & 1.56 \\
\hline 14 & 2 & 38.33471 & -121.67162 & 1.64 & 50 & 4 & 38.33159 & -121.67250 & 1.62 \\
\hline 15 & 2 & 38.33472 & -121.67156 & 1.60 & 51 & 4 & 38.33159 & -121.67244 & 1.59 \\
\hline 16 & 2 & 38.33473 & -121.67151 & 1.62 & 52 & 4 & 38.33159 & -121.67239 & 1.63 \\
\hline 17 & 2 & 38.33474 & -121.67146 & 1.62 & 53 & 4 & 38.33159 & -121.67233 & 1.63 \\
\hline 18 & 3 & 38.33014 & -121.67278 & 1.59 & 54 & 4 & 38.33159 & -121.67226 & 1.62 \\
\hline 19 & 3 & 38.33019 & -121.67280 & 1.62 & 55 & 4 & 38.33160 & -121.67221 & 1.63 \\
\hline 20 & 3 & 38.33023 & -121.67283 & 1.66 & 56 & 4 & 38.33131 & -121.67217 & 1.63 \\
\hline 21 & 3 & 38.33027 & -121.67285 & 1.67 & 57 & 4 & 38.33116 & -121.67214 & 1.57 \\
\hline 22 & 3 & 38.33032 & -121.67287 & 1.64 & 58 & 4 & 38.33083 & -121.67207 & 1.65 \\
\hline 23 & 3 & 38.33036 & -121.67289 & 1.67 & 59 & 4 & 38.33058 & -121.67216 & 1.60 \\
\hline 24 & 3 & 38.33040 & -121.67291 & 1.71 & 60 & 5 & 38.33391 & -121.67084 & 1.66 \\
\hline 25 & 3 & 38.33044 & -121.67294 & 1.70 & 61 & 5 & 38.33392 & -121.67077 & 1.54 \\
\hline 26 & 3 & 38.33048 & -121.67296 & 1.76 & 62 & 5 & 38.33392 & -121.67072 & 1.66 \\
\hline 27 & 3 & 38.33052 & -121.67298 & 1.69 & 63 & 5 & 38.33392 & -121.67067 & 1.67 \\
\hline 28 & 3 & 38.33056 & -121.67301 & 1.71 & 64 & 5 & 38.33393 & -121.67061 & 1.70 \\
\hline 29 & 3 & 38.33060 & -121.67303 & 1.70 & 65 & 5 & 38.33395 & -121.67056 & 1.66 \\
\hline 30 & 3 & 38.33065 & -121.67305 & 1.65 & 66 & 5 & 38.33398 & -121.67052 & 1.76 \\
\hline 31 & 3 & 38.33069 & -121.67308 & 1.64 & 67 & 5 & 38.33401 & -121.67048 & 1.80 \\
\hline 32 & 3 & 38.33072 & -121.67310 & 1.69 & 68 & 5 & 38.33405 & -121.67044 & 1.75 \\
\hline 33 & 3 & 38.33076 & -121.67312 & 1.66 & 69 & 5 & 38.33410 & -121.67039 & 1.74 \\
\hline 34 & 3 & 38.33080 & -121.67315 & 1.67 & 70 & 5 & 38.33413 & -121.67035 & 1.78 \\
\hline 35 & 3 & 38.33084 & -121.67318 & 1.68 & 71 & 5 & 38.33417 & -121.67032 & 1.83 \\
\hline 36 & 3 & 38.33088 & -121.67321 & 1.72 & 72 & 5 & 38.33419 & -121.67026 & 1.86 \\
\hline
\end{tabular}




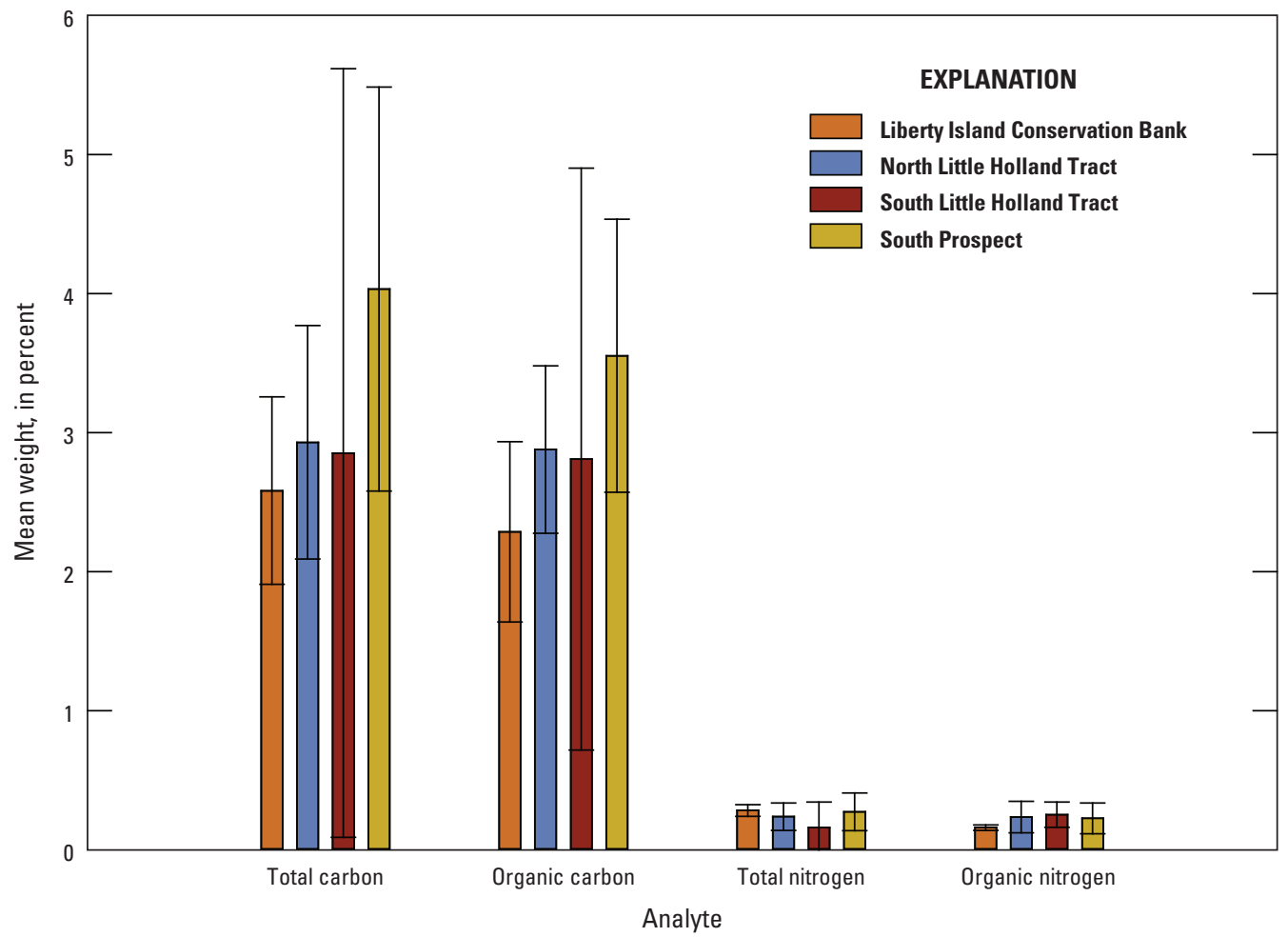

Figure 5. Mean weight as percentages of total carbon, organic carbon, total nitrogen, and total organic nitrogen measured in soil samples collected from four marsh sites in the Cache Slough region of the Sacramento-San Joaquin Delta, September 2014. Vertical bars represent plus and minus one standard deviation error. 
Table 6. Organic carbon and nitrogen measured in soil samples collected from marsh sites in the Cache Slough region of the Sacramento-San Joaquin Delta, September 2014.

[Numbers in brackets are U.S. Geological Survey (USGS) National Water Information System (NWIS) parameter codes. Abbreviations: hh:mm; hour:minute; mm/dd/yyyy; month/day/year; NAD 83, North American Datum of 1983]

\begin{tabular}{|c|c|c|c|c|c|c|c|c|}
\hline $\begin{array}{c}\text { USGS } \\
\text { station number }\end{array}$ & $\begin{array}{c}\text { USGS } \\
\text { station name }\end{array}$ & Marsh site & $\begin{array}{l}\text { Sample date } \\
\text { (mm/dd/yyyy) }\end{array}$ & $\begin{array}{c}\text { Sample } \\
\text { time } \\
\text { (hh:mm) }\end{array}$ & $\begin{array}{c}\text { Total } \\
\text { carbon } \\
\text { (percent) } \\
{[46247]}\end{array}$ & $\begin{array}{c}\text { Organic } \\
\text { carbon } \\
\text { (percent) } \\
{[01423]}\end{array}$ & $\begin{array}{c}\text { Total } \\
\text { nitrogen } \\
\text { (percent) } \\
{[01471]}\end{array}$ & $\begin{array}{c}\text { Organic } \\
\text { nitrogen } \\
\text { (percent) } \\
\text { [52760] }\end{array}$ \\
\hline 382006121401701 & Liberty Island Marsh Site 1 near Courtland, CA & $\begin{array}{l}\text { Liberty Island Conservation } \\
\text { Bank (LICB) }\end{array}$ & $09 / 23 / 2014$ & $12: 30$ & 1.90 & 1.68 & 0.27 & 0.16 \\
\hline 381955121401301 & Liberty Island Marsh Site 2 near Courtland, CA & $\begin{array}{l}\text { Liberty Island Conservation } \\
\text { Bank (LICB) }\end{array}$ & $09 / 23 / 2014$ & 13:05 & 3.25 & 2.97 & 0.33 & 0.18 \\
\hline 382006121401602 & Liberty Island Marsh Site 3 near Courtland, CA & $\begin{array}{l}\text { Liberty Island Conservation } \\
\text { Bank (LICB) }\end{array}$ & $09 / 23 / 2014$ & $13: 45$ & 2.60 & 2.21 & 0.25 & 0.14 \\
\hline 382005121392901 & Little Holland Tract Marsh Site 1 near Courtland, CA & North Little Holland Tract & $09 / 23 / 2014$ & $14: 20$ & 2.91 & 2.89 & 0.13 & 0.22 \\
\hline 382004121392801 & Little Holland Tract Marsh Site 2 near Courtland, CA & North Little Holland Tract & $09 / 23 / 2014$ & $16: 00$ & 2.10 & 2.27 & 0.26 & 0.13 \\
\hline 382007121392301 & Little Holland Tract Marsh Site 3 near Courtland, CA & North Little Holland Tract & $09 / 23 / 2014$ & $16: 15$ & 3.78 & 3.48 & 0.33 & 0.36 \\
\hline 381721121394701 & Prospect Slough Marsh Site 4 near Liberty Farms, CA & South Little Holland Tract & $09 / 23 / 2014$ & 11:05 & 1.68 & 1.98 & 0.32 & 0.35 \\
\hline 381718121395301 & Prospect Slough Marsh Site 5 near Liberty Farms, CA & South Little Holland Tract & $09 / 23 / 2014$ & $11: 25$ & 6.01 & 5.19 & 0.36 & 0.24 \\
\hline 381718121395201 & Prospect Slough Marsh Site 6 near Liberty Farms, CA & South Little Holland Tract & $09 / 23 / 2014$ & $11: 50$ & 0.87 & 1.26 & 0.12 & 0.17 \\
\hline 381414121403901 & Prospect Slough Marsh Site 1 near Rio Vista, CA & South Prospect & $09 / 23 / 2014$ & 09:15 & 2.70 & 2.60 & 0.14 & 0.11 \\
\hline 381416121403601 & Prospect Slough Marsh Site 2 near Rio Vista, CA & South Prospect & $09 / 23 / 2014$ & $09: 45$ & 5.58 & 4.56 & 0.41 & 0.33 \\
\hline 381420121403501 & Prospect Slough Marsh Site 3 near Rio Vista, CA & South Prospect & $09 / 23 / 2014$ & $10: 20$ & 3.82 & 3.50 & 0.27 & 0.24 \\
\hline
\end{tabular}




\section{Pesticide Analyses}

A total of 18 pesticides and degradates were detected in water samples. Five fungicides (azoxystrobin, boscalid, carbendazim, fluxapyroxad, and pyrimethanil), eight herbicides (clomazone, dithiopyr, diuron, fluridone, hexazinone, metolachlor, penoxsulam, and simazine), two herbicide degradates (3,4-DCA, and DCPMU), two insecticides (carbaryl and chlorantraniliprole), and one insecticide degradate (desulfinylfipronil) were detected (table 7). All water samples contained mixtures of 10-15 pesticides (fig. 6). Pesticide detection frequencies ranged from 7 to 100 percent, and eight pesticides (azoxystrobin, boscalid, clomazone, DCPMU, 3,4-DCA, diuron, hexazinone, and metolachlor) were detected in every water sample (table 8).

Pesticide concentrations ranged from below the method detection limits for some compounds up to 140.9 nanograms per liter (ng/L) for the fungicide azoxystrobin (table 7). Compounds detected at the highest concentrations included azoxystrobin and clomazone (two compounds applied to rice) as well as the herbicide hexazinone and the fungicide boscalid, both of which have a variety of agricultural applications. In general, insecticide concentrations were near or below method detection levels. Total detected pesticide mass per sample (the sum of all individual pesticide concentrations) ranged from 169.6 to $396.8 \mathrm{ng} / \mathrm{L}$. In general, herbicides and fungicides contributed nearly all of the total pesticide mass per sample. No pesticides were detected at concentrations greater than published Environmental Protection Agency aquatic-life benchmarks (U.S. Environmental Protection Agency, 2016).

The spatial distribution of pesticides was generally consistent among the five sites. In the few instances where a detected pesticide was not present at all five sites, the detections were generally at or below the respective method detection limits listed in table 2. Pesticide concentrations were also generally similar for the five sampling sites during a particular sampling date. In those cases in which a pesticide was detected at all five sampling sites during a particular site visit (32 instances), the relative standard deviation in concentration for the five sites sampled on a particular date ranged from 6 to 105 percent and averaged 26 percent. The highest concentration for a particular pesticide was most often (12 out of 32) detected at the Ulatis Creek input site (Cache Slough at Hass Slough near Liberty Farms), followed by the LICB restored marsh site ( 8 out of 32 ). No pesticides were detected in suspended sediment filtered from any of the water samples analyzed during this project.

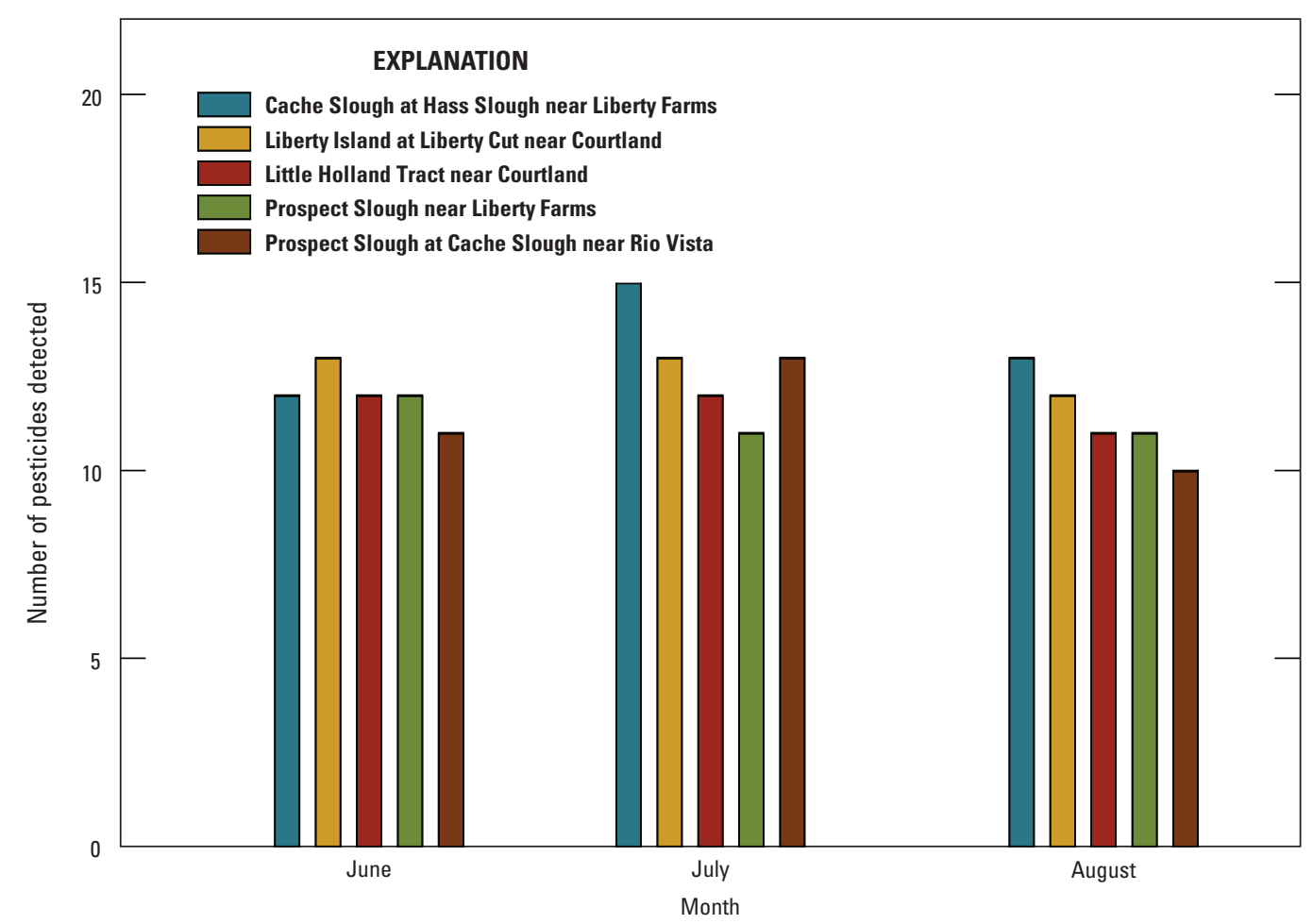

Figure 6. Number of current-use pesticides detected at five surface-water sites in the Cache Slough region of the Sacramento-San Joaquin Delta, 2015. 
Table 7. Pesticide concentrations measured in environmental water samples collected from the Cache Slough region of the Sacramento-San Joaquin Delta, California, 2015.

[Numbers in brackets are U.S. Geological Survey (USGS) National Water Information System (NWIS) parameter codes. Concentrations are in nanograms per liter. Results in parenthesis ( ) are below method detection limits and are estimates. Abbreviations: hh:mm, hours:minutes; —, not detected]

\begin{tabular}{|c|c|c|c|c|c|c|c|}
\hline $\begin{array}{l}\text { USGS station } \\
\text { number }\end{array}$ & USGS station name & Short site name & $\begin{array}{c}\text { Sample } \\
\text { time } \\
\text { (hh:mm) }\end{array}$ & $\begin{array}{l}\text { Azoxystrobin } \\
{[66589]}\end{array}$ & $\begin{array}{l}\text { Boscalid } \\
{[67550]}\end{array}$ & $\begin{array}{l}\text { Carbaryl } \\
\text { [65069] }\end{array}$ & $\begin{array}{l}\text { Carbendazim } \\
\text { [68548] }\end{array}$ \\
\hline \multicolumn{8}{|c|}{ Sample date: June 3, 2015} \\
\hline 381719121432301 & Cache Slough at Hass Slough near Liberty Farms, CA & Ulatis Creek & $10: 45$ & 77.9 & 29.0 & - & 4.2 \\
\hline 382006121401601 & Liberty Island at Liberty Cut near Courtland, CA & $\begin{array}{l}\text { Liberty Island Conservation } \\
\text { Bank (LICB) }\end{array}$ & $11: 30$ & 136.8 & 60.9 & 17.2 & 13.6 \\
\hline 382005121392801 & Little Holland Tract near Courtland, CA & North Little Holland Tract & $11: 50$ & 99.0 & 34.5 & - & 5.7 \\
\hline 381721121395301 & Prospect Slough near Liberty Farms, CA & South Little Holland Tract & 13:00 & 44.1 & 22.9 & - & 18.3 \\
\hline 381418121405301 & Prospect Slough at Cache Slough near Rio Vista, CA & South Prospect & $12: 35$ & 72.0 & 31.8 & - & - \\
\hline \multicolumn{8}{|c|}{ Sample date: July 6, 2015} \\
\hline 381719121432301 & Cache Slough at Hass Slough near Liberty Farms, CA & Ulatis Creek & $10: 40$ & 74.9 & 16.0 & - & 18.8 \\
\hline 382006121401601 & Liberty Island at Liberty Cut near Courtland, CA & $\begin{array}{l}\text { Liberty Island Conservation } \\
\text { Bank (LICB) }\end{array}$ & $11: 10$ & 50.3 & 18.8 & - & 12.2 \\
\hline 382005121392801 & Little Holland Tract near Courtland, CA & North Little Holland Tract & $11: 25$ & 55.1 & 16.0 & - & $(4.1)$ \\
\hline 381721121395301 & Prospect Slough near Liberty Farms, CA & South Little Holland Tract & $11: 45$ & 49.4 & 8.7 & - & - \\
\hline 381418121405301 & Prospect Slough at Cache Slough near Rio Vista, CA & South Prospect & $12: 10$ & 41.8 & 10.3 & - & 15.2 \\
\hline \multicolumn{8}{|c|}{ Sample date: August 13, 2015} \\
\hline 381719121432301 & Cache Slough at Hass Slough near Liberty Farms, CA & Ulatis Creek & $10: 30$ & 119.6 & 16.0 & - & 13.2 \\
\hline 382006121401601 & Liberty Island at Liberty Cut near Courtland, CA & $\begin{array}{l}\text { Liberty Island Conservation } \\
\text { Bank (LICB) }\end{array}$ & $11: 00$ & 112.5 & 25.3 & - & 6.2 \\
\hline 382005121392801 & Little Holland Tract near Courtland, CA & North Little Holland Tract & $11: 15$ & 121.7 & 25.2 & - & 11.0 \\
\hline 381721121395301 & Prospect Slough near Liberty Farms, CA & South Little Holland Tract & $11: 35$ & 140.9 & 20.8 & - & 15.3 \\
\hline 381418121405301 & Prospect Slough at Cache Slough near Rio Vista, CA & South Prospect & $11: 52$ & 137.2 & 14.0 & - & - \\
\hline
\end{tabular}


Table 7. Pesticide concentrations measured in environmental water samples collected from the Cache Slough region of the Sacramento-San Joaquin Delta, California, 2015.Continued

[Numbers in brackets are U.S. Geological Survey (USGS) National Water Information System (NWIS) parameter codes. Concentrations are in nanograms per liter. Results in parenthesis ( ) are below method detection limits and are estimates. Abbreviations: hh:mm, hours:minutes; —, not detected]

\begin{tabular}{|c|c|c|c|c|c|c|c|c|}
\hline $\begin{array}{l}\text { USGS station } \\
\text { number }\end{array}$ & USGS station name & Short site name & $\begin{array}{c}\text { Sample } \\
\text { time } \\
\text { (hh:mm) }\end{array}$ & $\begin{array}{c}\text { Chlorantraniliprole } \\
{[51856]}\end{array}$ & $\begin{array}{c}\text { Clomazone } \\
\text { [67562] }\end{array}$ & $\begin{array}{l}\text { DCPMU } \\
\text { [68231] }\end{array}$ & $\begin{array}{c}\text { 3,4-DCA } \\
{[66584]}\end{array}$ & $\begin{array}{c}\text { Dithiopyr } \\
\text { [51837] }\end{array}$ \\
\hline \multicolumn{9}{|c|}{ Sample date: June 3, 2015} \\
\hline 381719121432301 & Cache Slough at Hass Slough near Liberty Farms, CA & Ulatis Creek & $10: 45$ & - & 56.9 & 17.1 & 6.5 & - \\
\hline 382006121401601 & Liberty Island at Liberty Cut near Courtland, CA & $\begin{array}{l}\text { Liberty Island Conservation } \\
\text { Bank (LICB) }\end{array}$ & $11: 30$ & - & 26.1 & $(2.8)$ & 5.8 & - \\
\hline 382005121392801 & Little Holland Tract near Courtland, CA & North Little Holland Tract & $11: 50$ & - & 31.5 & 4.2 & 11.2 & - \\
\hline 381721121395301 & Prospect Slough near Liberty Farms, CA & South Little Holland Tract & 13:00 & - & 75.6 & 3.6 & 6.1 & - \\
\hline 381418121405301 & Prospect Slough at Cache Slough near Rio Vista, CA & South Prospect & $12: 35$ & - & 59.7 & $(2.1)$ & 6.6 & - \\
\hline \multicolumn{9}{|c|}{ Sample date: July 6, 2015} \\
\hline 381719121432301 & Cache Slough at Hass Slough near Liberty Farms, CA & Ulatis Creek & $10: 40$ & $(3.7)$ & 45.1 & 4.6 & 12.0 & - \\
\hline 382006121401601 & Liberty Island at Liberty Cut near Courtland, CA & $\begin{array}{l}\text { Liberty Island Conservation } \\
\text { Bank (LICB) }\end{array}$ & $11: 10$ & 4.0 & 34.6 & 4.2 & 7.8 & - \\
\hline 382005121392801 & Little Holland Tract near Courtland, CA & North Little Holland Tract & $11: 25$ & $(3.5)$ & 31.9 & 4.4 & 10.5 & - \\
\hline 381721121395301 & Prospect Slough near Liberty Farms, CA & South Little Holland Tract & $11: 45$ & (3.9) & 37.0 & 4.6 & 16.2 & - \\
\hline 381418121405301 & Prospect Slough at Cache Slough near Rio Vista, CA & South Prospect & $12: 10$ & $(3.4)$ & 31.1 & $(3.2)$ & 14.4 & - \\
\hline \multicolumn{9}{|c|}{ Sample date: August 13, 2015} \\
\hline 381719121432301 & Cache Slough at Hass Slough near Liberty Farms, CA & Ulatis Creek & $10: 30$ & 6.9 & 7.0 & $(3.2)$ & 5.0 & 2.0 \\
\hline 382006121401601 & Liberty Island at Liberty Cut near Courtland, CA & $\begin{array}{l}\text { Liberty Island Conservation } \\
\text { Bank (LICB) }\end{array}$ & 11:00 & $(3.0)$ & 6.3 & $(2.3)$ & 5.9 & - \\
\hline 382005121392801 & Little Holland Tract near Courtland, CA & North Little Holland Tract & $11: 15$ & - & 7.0 & $(3.4)$ & 6.5 & - \\
\hline 381721121395301 & Prospect Slough near Liberty Farms, CA & South Little Holland Tract & $11: 35$ & - & 7.4 & $(3.2)$ & 6.2 & - \\
\hline 381418121405301 & Prospect Slough at Cache Slough near Rio Vista, CA & South Prospect & $11: 52$ & - & 6.8 & $(2.0)$ & 7.7 & - \\
\hline
\end{tabular}


Table 7. Pesticide concentrations measured in environmental water samples collected from the Cache Slough region of the Sacramento-San Joaquin Delta, California, 2015.Continued

[Numbers in brackets are U.S. Geological Survey (USGS) National Water Information System (NWIS) parameter codes. Concentrations are in nanograms per liter. Results in parenthesis ( ) are below method detection limits and are estimates. Abbreviations: hh:mm, hours:minutes; —, not detected]

\begin{tabular}{|c|c|c|c|c|c|c|c|}
\hline $\begin{array}{l}\text { USGS station } \\
\text { number }\end{array}$ & USGS station name & Short site name & $\begin{array}{c}\text { Sample } \\
\text { time } \\
\text { (hh:mm) }\end{array}$ & $\begin{array}{l}\text { Diuron } \\
{[66598]}\end{array}$ & $\begin{array}{c}\text { Desulfinylfipronil } \\
{[66607]}\end{array}$ & $\begin{array}{c}\text { Fluridone } \\
\text { [51864] }\end{array}$ & $\begin{array}{c}\text { Fluxapyroxad } \\
\text { [51851] }\end{array}$ \\
\hline \multicolumn{8}{|c|}{ Sample date: June 3, 2015} \\
\hline 381719121432301 & Cache Slough at Hass Slough near Liberty Farms, CA & Ulatis Creek & $10: 45$ & 45.3 & - & 6.1 & 5.3 \\
\hline 382006121401601 & Liberty Island at Liberty Cut near Courtland, CA & $\begin{array}{l}\text { Liberty Island Conservation } \\
\text { Bank (LICB) }\end{array}$ & $11: 30$ & 8.4 & - & 3.9 & 13.6 \\
\hline 382005121392801 & Little Holland Tract near Courtland, CA & North Little Holland Tract & $11: 50$ & 13.6 & - & 5.1 & 6.2 \\
\hline 381721121395301 & Prospect Slough near Liberty Farms, CA & South Little Holland Tract & 13:00 & 10.1 & - & 5.4 & 5.3 \\
\hline 381418121405301 & Prospect Slough at Cache Slough near Rio Vista, CA & South Prospect & $12: 35$ & 10.9 & - & 5.2 & 5.9 \\
\hline \multicolumn{8}{|c|}{ Sample date: July 6, 2015} \\
\hline 381719121432301 & Cache Slough at Hass Slough near Liberty Farms, CA & Ulatis Creek & $10: 40$ & 7.3 & 3.8 & 3.7 & - \\
\hline 382006121401601 & Liberty Island at Liberty Cut near Courtland, CA & $\begin{array}{l}\text { Liberty Island Conservation } \\
\text { Bank (LICB) }\end{array}$ & $11: 10$ & 6.2 & - & $(3.0)$ & - \\
\hline 382005121392801 & Little Holland Tract near Courtland, CA & North Little Holland Tract & $11: 25$ & 7.3 & - & 3.7 & - \\
\hline 381721121395301 & Prospect Slough near Liberty Farms, CA & South Little Holland Tract & $11: 45$ & 9.8 & - & 6.1 & - \\
\hline 381418121405301 & Prospect Slough at Cache Slough near Rio Vista, CA & South Prospect & $12: 10$ & 5.8 & - & 4.2 & - \\
\hline \multicolumn{8}{|c|}{ Sample date: August 13, 2015} \\
\hline 381719121432301 & Cache Slough at Hass Slough near Liberty Farms, CA & Ulatis Creek & $10: 30$ & 4.5 & 2.5 & - & $(3.6)$ \\
\hline 382006121401601 & Liberty Island at Liberty Cut near Courtland, CA & $\begin{array}{l}\text { Liberty Island Conservation } \\
\text { Bank (LICB) }\end{array}$ & $11: 00$ & 3.2 & 2.6 & - & $(4.4)$ \\
\hline 382005121392801 & Little Holland Tract near Courtland, CA & North Little Holland Tract & $11: 15$ & 3.6 & 2.7 & - & $(4.6)$ \\
\hline 381721121395301 & Prospect Slough near Liberty Farms, CA & South Little Holland Tract & $11: 35$ & 4.1 & 3.0 & - & $(4.4)$ \\
\hline 381418121405301 & Prospect Slough at Cache Slough near Rio Vista, CA & South Prospect & $11: 52$ & 4.1 & 2.6 & - & $(3.6)$ \\
\hline
\end{tabular}


Table 7. Pesticide concentrations measured in environmental water samples collected from the Cache Slough region of the Sacramento-San Joaquin Delta, California, 2015.Continued

[Numbers in brackets are U.S. Geological Survey (USGS) National Water Information System (NWIS) parameter codes. Concentrations are in nanograms per liter. Results in parenthesis ( ) are below method detection limits and are estimates. Abbreviations: hh:mm, hours:minutes; —, not detected]

\begin{tabular}{|c|c|c|c|c|c|c|c|c|}
\hline $\begin{array}{l}\text { USGS station } \\
\text { number }\end{array}$ & USGS station name & Short site name & $\begin{array}{c}\text { Sample } \\
\text { time } \\
\text { (hh:mm) }\end{array}$ & $\begin{array}{c}\text { Hexazinone } \\
\text { [65085] }\end{array}$ & $\begin{array}{c}\text { Metolachlor } \\
\text { [65090] }\end{array}$ & $\begin{array}{c}\text { Penoxsulam } \\
{[51863]}\end{array}$ & $\begin{array}{c}\text { Pyrimeth- } \\
\text { anil } \\
\text { [67717] }\end{array}$ & $\begin{array}{c}\text { Simazine } \\
\text { [65105] }\end{array}$ \\
\hline \multicolumn{9}{|c|}{ Sample date: June 3, 2015} \\
\hline 381719121432301 & Cache Slough at Hass Slough near Liberty Farms, CA & Ulatis Creek & $10: 45$ & 99.4 & 29.6 & - & - & 6.5 \\
\hline 382006121401601 & Liberty Island at Liberty Cut near Courtland, CA & $\begin{array}{l}\text { Liberty Island Conservation } \\
\text { Bank (LICB) }\end{array}$ & $11: 30$ & 76.8 & 23.6 & - & - & 7.3 \\
\hline 382005121392801 & Little Holland Tract near Courtland, CA & North Little Holland Tract & $11: 50$ & 56.5 & 22.1 & - & - & 6.9 \\
\hline 381721121395301 & Prospect Slough near Liberty Farms, CA & South Little Holland Tract & $13: 00$ & 45.2 & 22.6 & - & - & 6.0 \\
\hline 381418121405301 & Prospect Slough at Cache Slough near Rio Vista, CA & South Prospect & $12: 35$ & 53.7 & 24.6 & - & - & 6.2 \\
\hline \multicolumn{9}{|c|}{ Sample date: July 6, 2015} \\
\hline 381719121432301 & Cache Slough at Hass Slough near Liberty Farms, CA & Ulatis Creek & $10: 40$ & 27.6 & 15.3 & 4.3 & 5.5 & $(2.6)$ \\
\hline 382006121401601 & Liberty Island at Liberty Cut near Courtland, CA & $\begin{array}{l}\text { Liberty Island Conservation } \\
\text { Bank (LICB) }\end{array}$ & $11: 10$ & 24.0 & 11.2 & 4.4 & $(2.6)$ & - \\
\hline 382005121392801 & Little Holland Tract near Courtland, CA & North Little Holland Tract & $11: 25$ & 19.8 & 9.4 & - & $(3.9)$ & - \\
\hline 381721121395301 & Prospect Slough near Liberty Farms, CA & South Little Holland Tract & $11: 45$ & 19.3 & 15.8 & - & 4.8 & - \\
\hline 381418121405301 & Prospect Slough at Cache Slough near Rio Vista, CA & South Prospect & $12: 10$ & 17.5 & 13.1 & 5.4 & 5.4 & - \\
\hline \multicolumn{9}{|c|}{ Sample date: August 13, 2015} \\
\hline 381719121432301 & Cache Slough at Hass Slough near Liberty Farms, CA & Ulatis Creek & $10: 30$ & 29.6 & 8.7 & - & - & - \\
\hline 382006121401601 & Liberty Island at Liberty Cut near Courtland, CA & $\begin{array}{l}\text { Liberty Island Conservation } \\
\text { Bank (LICB) }\end{array}$ & 11:00 & 34.8 & 6.6 & - & - & - \\
\hline 382005121392801 & Little Holland Tract near Courtland, CA & North Little Holland Tract & $11: 15$ & 33.8 & 7.5 & - & - & - \\
\hline 381721121395301 & Prospect Slough near Liberty Farms, CA & South Little Holland Tract & $11: 35$ & 32.9 & 8.5 & - & - & - \\
\hline 381418121405301 & Prospect Slough at Cache Slough near Rio Vista, CA & South Prospect & $11: 52$ & 27.0 & 8.5 & - & - & - \\
\hline
\end{tabular}


Table 8. Detection frequencies of pesticides in water samples collected from the Cache Slough region of the Sacramento-San Joaquin Delta, California, 2015.

\begin{tabular}{lcc}
\hline \multicolumn{1}{c}{ Pesticide } & $\begin{array}{c}\text { Pesticide } \\
\text { type }\end{array}$ & $\begin{array}{c}\text { Detection frequency } \\
\text { (percent) }\end{array}$ \\
\hline Azoxystrobin & Fungicide & 100 \\
Boscalid & Fungicide & 100 \\
Clomazone & Herbicide & 100 \\
$N$-(3,4-Dichlorophenyl)- $N$ ' & Herbicide & 100 \\
$\quad$ methylurea (DCPMU) & degradate & \\
3,4-Dichloroaniline & Herbicide & 100 \\
$\quad$ (3,4-DCA) & degradate & \\
Diuron & Herbicide & 100 \\
Hexazinone & Herbicide & 100 \\
Metolachlor & Herbicide & 100 \\
Carbendazim & Fungicide & 80 \\
Fluridone & Herbicide & 67 \\
Fluxapyroxad & Fungicide & 67 \\
Chlorantraniliprole & Insecticide & 47 \\
Desulfinylfipronil & Insecticide & 40 \\
& degradate & \\
Simazine & Herbicide & 40 \\
Pyrimethanil & Fungicide & 33 \\
Penoxsulam & Herbicide & 20 \\
Carbaryl & Insecticide & 7 \\
Dithiopyr & Herbicide & 7 \\
\hline & & \\
\hline
\end{tabular}

A comparison of pesticide detections for only the restored and remnant natural marsh sites (Ulatis Creek input-site data excluded) showed that the number of pesticides detected during a particular sampling was greatest at the LICB during June and August and tied for greatest during July (fig. 6). A similar comparison of total pesticide concentrations showed that concentrations were highest at the LICB in June and July compared to the natural marsh sites, but were lower at the LICB than at the natural marsh sites in August (table 7). These differences do not in and of themselves support the hypothesis that pesticide contamination is limiting plant growth in the LICB site. Further research is needed to determine whether these compounds could be affecting plant productivity at the LICB, particularly because 2015 was a drought year, when less rice was planted than usual (U.S. Department of Agriculture, 2017), and therefore, overall pesticide use in the region was also likely less than usual.

\section{Conclusions}

The Liberty Island Conservation Bank (LICB) contains vegetation that is of short stature and chlorotic relative to neighboring marshes in the Cache Slough region. This study tested whether (1) improper grading of the marsh plain, (2) contamination by pesticides from urban and agricultural sources upstream from the site, or (3) nitrogen-deficient soil could be responsible for these differences. The data showed no differences in total or organic carbon and nitrogen in the surface-soil layers among the marshes. Although we did not sample the full soil profile, the results indicated that differences among soils were not likely to be the main reason for the short and chlorotic vegetation at the LICB. An analysis of pesticides in water and suspended sediments collected during the summer of 2015 showed no major differences among sites. Because 2015 was a drought year, however, less rice than usual was planted, likely resulting in less pesticide use in the region compared with prior years. More research needs to be done to determine whether pesticides could be affecting plant growth at the LICB.

Lastly, an RTK-GPS elevation survey was carried out to determine whether there is an elevational difference between the constructed marsh at LICB and a natural marsh, also in the northern Cache Slough region (north Little Holland Tract), that has similar hydrodynamics. The results of the survey showed that, on average, the marsh plain is 26 centimeters higher at the LICB than at the north Little Holland Tract marsh. This result indicates that the higher elevation of the marsh plain at the LICB is altering its hydroperiod, resulting in decreased flooding and leading to reduced growth and vigor of marsh vegetation. Although the LICB cannot be regraded at this point without great expense, it could be possible to reduce the sharp angle of the marsh edge to facilitate tidal flooding on the marsh periphery. Grading the marsh plain at the correct elevation range is necessary for the establishment of marsh vegetation and the development of the full suite of ecosystem services that marshes provide. A better system of tidal benchmarks and more research on the elevation range required for successful colonization of herbaceous and scrubshrub wetlands in the delta is needed to improve the success of wetland restoration in the region. 


\section{References Cited}

California Department of Pesticide Regulation, 2015, Pesticide use report for 2014, digital data accessed March 6, 2016, at http://www.cdpr.ca.gov/docs/pur/purmain.htm.

Hladik, Michelle, and Calhoun, D.L., 2012, Analysis of the herbicide diuron, three diuron degradates, and six neonicotinoid insecticides in water-Method details and application to two Georgia streams: U.S. Geological Survey Scientific Investigations Report 2012-5206, 10 p., https://pubs.er.usgs.gov/publication/sir20125206.

Hladik, M.L., and McWayne, M.M., 2012, Methods of analysis - Determination of pesticides in sediment using gas chromatography/mass spectrometry: U.S. Geological Survey Techniques and Methods 5-C3, 18 p., http://pubs.usgs.gov/tm/tm5c3.

Hladik, M.L., Smalling, K.L., and Kuivila, K.M., 2008, A multi-residue method for the analysis of pesticides and pesticide degradates in water using HLB solidphase extraction and gas chromatography-ion trap mass spectrometry: Bulletin of Environmental Contamination and Toxicology, v. 80, no. 2, p. 139-144, http://dx.doi.org/10.1007/s00128-007-9332-2.

Mitsch, W.J., and Gosselink, J.G., 2000, Wetlands (3d ed.): New York, N.Y., John Wiley and Sons, Inc., 456 p.

Orlando, J.L., Jacobson, L.A., and Kuivila, Kathryn, 2004, Dissolved pesticide and organic carbon concentrations detected in surface waters, Northern Central Valley, California, 2001-2002: U.S. Geological Survey OpenFile Report 2004-1214, 40 p., https://pubs.er.usgs.gov/publication/ofr20041214.

Pilbeam, D.J., 2015, Chapter 2: Nitrogen in Barker, A.V., and Pilbeam, D.J., eds., Handbook of plant nutrition ( $2 \mathrm{~d}$ ed.): Boca Raton, Fla., CRC Press, 773 p.
Sloey, T.M., and Hester, M.W., 2016, Interactions between soil physicochemistry and belowground biomass production in a freshwater tidal marsh: Plant and Soil, v. 401, nos. 1-2, p. 397-408, http://dx.doi.org/10.1007/s11104-015-2760-6.

Swanson, K.M., Drexler, J.Z., Fuller, C.C., and Schoellhamer, D.H., 2015, Modeling tidal freshwater marsh sustainability in the Sacramento-San Joaquin Delta under a broad suite of potential future scenarios: San Francisco Estuary and Watershed Science, v. 13, no. 1, 21 p., http://escholarship.org/uc/item/9h8197nt\#page-1.

Weston, D.P., and Lydy, M.J., 2010, Urban and agricultural sources of pyrethroid insecticides to the Sacramento-San Joaquin Delta of California: Environmental Science and Technology, v. 44, no. 5, p. 1833-1840, http://pubs.acs.org/doi/abs/10.1021/es9035573.

Wildlands, Inc., 2009, Liberty Island Conservation Bank initial study/mitigated negative declaration: Rocklin, Calif., 110 p., http://www.deltarevision.com/2009_even_more_ docs/LibertyIsland_April09_with\%20tabloid\%20figures. pdf.

U.S. Department of Agriculture, 2017, California rice county estimates, accessed June 5, 2017, at https:/www.nass.usda. gov/Statistics_by_State/California/Publications/County_ Estimates/2016/201703RICECNTY.pdf.

U.S. Environmental Protection Agency, 2016, Aquatic life benchmarks for pesticide registration, accessed March 24, 2016, at https:/www.epa.gov/pesticide-science-andassessing-pesticide-risks/aquatic-life-benchmarks-pesticideregistration.

Zimmerman, C.F., Keefe, C.W., and Bashe, Jerry, 1997, Determination of carbon and nitrogen in sediments and particulates of estuarine/coastal waters using elemental analysis, method 440.0, rev. 1.4: U.S. Environmental Protection Agency, accessed September 14, 2012, at http:// www.caslab.com/EPA-Methods/PDF/EPA-Method-440.pdf. 

Publishing support provided by the U.S. Geological Survey Science Publishing Network, Sacramento, Publishing Service Center

For more information concerning the research in this report, contact the Director, California Water Science Center U.S. Geological Survey 6000 J Street, Placer Hall 


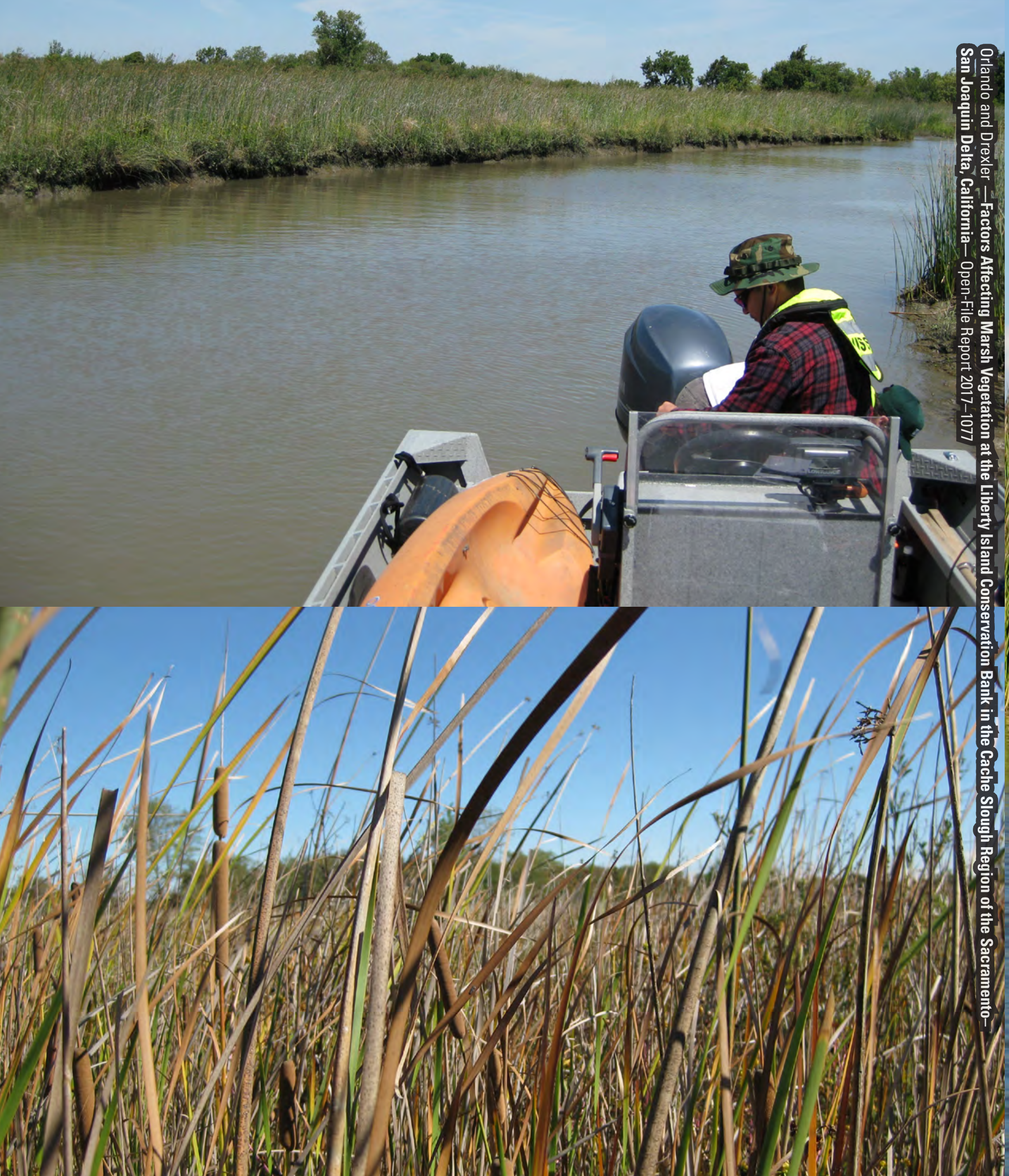

